\title{
A DETAILED MODEL ATMOSPHERE ANALYSIS OF COOL WHITE DWARFS IN THE SLOAN DIGITAL SKY SURVEY*
}

\author{
Mukremin Kilic $^{1,9}$, S. K. LeggetT ${ }^{2}$, P.-E. Tremblay ${ }^{3}$, Ted von Hippel ${ }^{4}$, P. Bergeron ${ }^{3}$, Hugh C. Harris ${ }^{5}$, \\ Jeffrey A. MunN ${ }^{5}$, Kurtis A. Williams ${ }^{6}$, Evalyn Gates ${ }^{7}$, and J. Farihi ${ }^{8}$ \\ ${ }^{1}$ Smithsonian Astrophysical Observatory, 60 Garden Street, Cambridge, MA 02138, USA; mkilic@ cfa.harvard.edu \\ ${ }^{2}$ Gemini Observatory, 670 N. A'ohoku Place, Hilo, HI 96720, USA \\ ${ }^{3}$ Département de Physique, Université de Montréal, C.P. 6128, Succursale Centre-Ville, Montréal, Québec H3C 3J7, Canada \\ ${ }^{4}$ Physics Department, Siena College, 515 Loudon Road, Loudonville, NY 12211, USA \\ ${ }^{5}$ U.S. Naval Observatory, Flagstaff Station, 10391 W. Naval Observatory Road, Flagstaff, AZ 86001, USA \\ ${ }^{6}$ Department of Astronomy, 1 University Station C1400, Austin, TX 78712, USA \\ ${ }^{7}$ Kavli Institute for Cosmological Physics and Department of Astronomy \& Astrophysics, The University of Chicago, \\ 5640 South Ellis Avenue, Chicago, IL 60637, USA \\ ${ }^{8}$ Department of Physics \& Astronomy, University of Leicester, Leicester LE1 7RH, UK \\ Received 2010 April 20; accepted 2010 July 15; published 2010 August 26
}

\begin{abstract}
We present optical spectroscopy and near-infrared photometry of 126 cool white dwarfs (WDs) in the Sloan Digital Sky Survey (SDSS). Our sample includes high proper motion targets selected using the SDSS and USNOB astrometry and a dozen previously known ultracool WD candidates. Our optical spectroscopic observations demonstrate that a clean selection of large samples of cool WDs in the SDSS (and the SkyMapper, Pan-STARRS, and the Large Synoptic Survey Telescope data sets) is possible using a reduced proper motion diagram and a tangential velocity cut-off (depending on the proper motion accuracy) of $30 \mathrm{~km} \mathrm{~s}^{-1}$. Our near-infrared observations reveal eight new stars with significant absorption. We use the optical and near-infrared photometry to perform a detailed model atmosphere analysis. More than $80 \%$ of the stars in our sample are consistent with either pure hydrogen or pure helium atmospheres. However, the eight stars with significant infrared absorption and the majority of the previously known ultracool WD candidates are best explained with mixed hydrogen and helium atmosphere models. The age distribution of our sample is consistent with a Galactic disk age of 8 Gyr. A few ultracool WDs may be as old as 12-13 Gyr, but our models have problems matching the spectral energy distributions of these objects. There are only two halo WD candidates in our sample. However, trigonometric parallax observations are required for accurate mass and age determinations and to confirm their membership in the halo.
\end{abstract}

Key words: stars: atmospheres - stars: evolution - white dwarfs

Online-only material: color figures

\section{INTRODUCTION}

White dwarf (WD) cosmochronology offers an independent age dating method for the Galactic disk and halo. WDs are initially hot and consequently cool rapidly, though the cooling rate slows as their temperature drops, allowing the oldest WDs to remain visible. Because the cooling rate slows, any census finds more and more WDs at lower and lower temperatures until, quite abruptly, we find no more of them. The position of this cut-off in temperature (and luminosity) is directly related to the age of the system. Starting with Winget et al. (1987) and Liebert et al. (1988) numerous studies targeted the oldest WDs in the Galactic disk in order to derive an accurate age. The current best estimate for the age of the Galactic disk based on WDs comes from Leggett et al. (1998), who performed a detailed model atmosphere analysis of the optical and infrared spectral energy distributions (SEDs) of 43 WDs to derive an age of $8 \pm 1.5 \mathrm{Gyr}$.

Within the last decade, the Sloan Digital Sky Survey (SDSS) emerged as the main resource for WD studies. The SDSS provides an unprecedented opportunity to increase the cool WD sample size from a few dozen to hundreds or thousands, and also extend the age dating method to the thick disk and halo. Kleinman et al. (2004) and Eisenstein et al. (2006) have

\footnotetext{
* Based on observations obtained at the Hobby-Eberly Telescope (HET), Gemini Observatory, and the Infrared Telescope Facility (IRTF).

9 Spitzer Fellow
}

already discovered over 9000 WDs in the SDSS Data Release 4 area. However, the majority of these objects are relatively warm. WDs cooler than about $7000 \mathrm{~K}$ are buried in the stellar color-color loci (Kilic et al. 2004) and they cannot be identified based on photometry alone. Proper motion offers an efficient means to delineate cooler WDs from the much larger number of main-sequence stars. Reduced proper motion, defined as $H=m+5 \log \mu+5=M+5 \log V_{\tan }-3.379$, has long been used as a proxy for absolute magnitude for samples with similar kinematics. Munn et al. (2004) used the SDSS and USNO-B (five epochs; Monet et al. 2003) astrometry to derive

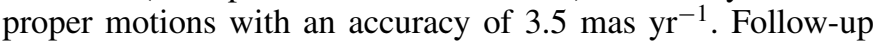
spectroscopy of high proper motion targets at the MMT, HET, and the McDonald $2.7 \mathrm{~m}$ telescope showed that WDs occupy a locus in the reduced proper motion diagram cleanly separated from most subdwarfs and that samples of WDs can be defined using this diagram with contamination by subdwarfs and quasars of only a few percent (Kilic et al. 2006a). Harris et al. (2006) used this result to create statistically complete catalogs of WDs and published a substantially improved disk luminosity function including $6000 \mathrm{WD}$ candidates. This luminosity function is consistent with the Leggett et al. (1998) result, but the lack of infrared photometry prevented Harris et al. (2006) from a definite conclusion about the implied age. Collision-induced opacity due to molecular hydrogen becomes important in the high-density atmospheres of cool WDs that contain even small 
traces of hydrogen. This opacity dominates in the near-infrared, and without near-infrared photometry the surface compositions and temperatures of WDs cooler than about $5000 \mathrm{~K}$ are uncertain.

In order to improve the cool end of the luminosity function presented by Harris et al. (2006) we obtained optical spectroscopy and/or near-infrared photometry of 156 of the coolest WDs. Our near-infrared photometry sample includes 126 stars. Our target selection criteria and observations are discussed in Section 2, while an analysis of the observational data and results from this analysis are presented in Section 3. Various implications of these results are then discussed in Sections 4 and 5.

\section{TARGET SELECTION AND OBSERVATIONS}

Based on a reduced proper motion diagram for the SDSS Data Release 3 footprint, Harris et al. (2006) derive cool WD samples by taking all stars below and blueward of the WD model curves for $V_{\tan }=20,30$, and $40 \mathrm{~km} \mathrm{~s}^{-1}$. They use the Bergeron et al. (1995) models to fit all five SDSS magnitudes to determine temperature, distance, bolometric magnitude, and tangential velocity for each star. The choice of hydrogen or helium atmosphere models has little effect on the estimated $M_{\text {bol }}$ for relatively warm WDs. However, the colors are significantly different for pure hydrogen and pure helium atmosphere models for stars fainter than $M_{\mathrm{bol}} \approx 14.6\left(T_{\mathrm{eff}} \approx 5300 \mathrm{~K}\right)$. Lacking infrared data, Harris et al. (2006) make a weighted $\mathrm{H} / \mathrm{He}$ assignment for each star based on the fraction of each type from the studies in the literature. Adopting a higher or lower fraction of hydrogen-dominated stars changes the faint end of the luminosity function significantly (see their Figure 7). Therefore, the exact luminosity of the cut-off in the luminosity function, the shape of the drop, and the extent of the faint tail are uncertain based on the SDSS data only. We target all WDs with $M_{\text {bol }}>14.6$ from the $V_{\text {tan }} \geqslant 20 \mathrm{~km} \mathrm{~s}^{-1}$ sample of Harris et al. (2006) for follow-up optical spectroscopy and near-infrared photometric observations. In addition, we target all 13 ultracool WD candidates identified by Gates et al. (2004) and Harris et al. (2008; see also Vidrih et al. 2007). Our goal is to create a clean sample of WDs that can be used to improve the faint end of the luminosity function.

\subsection{Optical Spectroscopy}

We obtained low-resolution spectroscopy of 98 targets using the $9.2 \mathrm{~m}$ HET in the queue mode and the Marcario Low Resolution Spectrograph (LRS; Hill et al. 1998). Our observations were performed between 2004 October and 2006 July. We used a 1".5 slit, Grism 2, and the GG385 blocking filter (with $50 \%$ transmission cut-on at $385 \mathrm{~nm}$ ) to obtain spectra with a wavelength coverage of 4280-7340 $\AA$ and a spectral resolution of $6 \AA$. A spectrophotometric standard star was observed each night for flux calibration (as part of the queue), and $\mathrm{Ne}-\mathrm{Cd}$ wavelength calibration lamp exposures were obtained after each science exposure. The data were reduced using standard IRAF ${ }^{10}$ routines.

\subsection{Near-infrared Photometry}

We obtained $J H K$ photometry of 126 WDs using the Near Infra-Red Imager and Spectrometer (NIRI) on Gemini-North,

\footnotetext{
10 IRAF is distributed by the National Optical Astronomy Observatory, which is operated by the Association of Universities for Research in Astronomy, Inc., under cooperative agreement with the National Science Foundation.
}

the 0.8-5.4 $\mu \mathrm{m}$ medium-resolution spectrograph and imager (SpeX) on the Infrared Telescope Facility (IRTF), and the WideField Camera (WFCAM) on the United Kingdom Infra-Red Telescope (UKIRT). The Gemini observations were obtained as part of the queue programs GN-2005B-Q-33, GN-2006A-Q-69, and GN-2008A-Q-78. The IRTF observations were obtained on several observing runs between 2004 December and 2006 April. The UKIRT observations (of J0146+1404 and J2239+0018) were performed on UT 2007 September 20 under the service program U/SERV/1762. The field of view of the NIRI observations is either $51^{\prime \prime} \times 51^{\prime \prime}$ or $120^{\prime \prime} \times 120^{\prime \prime}$ and that of the SpeX is $60^{\prime \prime} \times 60^{\prime \prime}$. We used a five or nine position dither pattern with 25-60 s exposures. We used the Gemini and NIRI packages in IRAF to reduce the data and the UKIRT faint standards (Leggett et al. 2006) to calibrate the photometry. The WFCAM images are processed through the UKIRT Infrared Deep Sky Survey (UKIDSS; Lawrence et al. 2007) pipeline. We use the merged source catalogs from the WFCAM science archive for our service program. The derived magnitudes are in the Mauna Kea photometric system (Tokunaga \& Vacca 2005). If available, we use observations of several standard stars in a single night to estimate the errors in nightly zero points. The typical errors in our photometry (including the nightly zero point errors of 0.01-0.02 mag) are about $0.04 \mathrm{mag}$.

\subsubsection{NIRI Nonlinearity Issues}

NIRI suffers from nonlinearities that are correctable. The detector response is a function of the counts in the pixel, the exposure time, the read mode, the bias level, and the vertical position on the detector. Using NIRI spectroscopic flats, A. Stephens developed and kindly made available to us a Python script that calculates and applies a per-pixel linearity correction for NIRI data. We use the 2008 April version of this script to correct our data. Without this correction, the photometry is $10 \%-15 \%$ fainter than expected.

Eighteen of our targets are detected in the UKIDSS, at least in one band. In order to make sure that our photometric reductions are reliable, we compare our NIRI photometry with the UKIDSS photometry. Our results are consistent with the UKIDSS results within the errors. However, NIRI photometry is on average fainter by $0.016,0.022$, and $0.024 \mathrm{mag}$ in $J, H$, and $K$ filters, respectively. We correct for this systematic effect by making our photometry brighter by the above amounts. The corrected photometry is well within the errors in our original measurements.

\section{RESULTS}

The optical spectra for the newly observed WDs at the HET are shown in Figures 1-5. Our targets include 29 DAs that show $\mathrm{H} \alpha$ absorption (Figure 1; see the spectral classification system of Sion et al. 1983), 35 DCs with featureless spectra (Figure 2), 4 DQs with molecular carbon bands (+2 DQs with SDSS spectra; Figure 3), 2 DZAs with magnesium, sodium, and hydrogen lines (Figure 4), and 2 unresolved DA + dM (M dwarf) pairs (Figure 5). Most spectra show sky subtraction problems at 5577, 5890/5896, and $6300 \AA$.

The spectra presented in Figure 2 seem featureless. However, weak $\mathrm{H} \alpha$ absorption may be hidden in the noise, and higher resolution and higher signal-to-noise ratio observations may reveal weak $\mathrm{H} \alpha$ for some of the targets in this figure.

Two of the stars in our sample, J1247+0646 and J1442+4013, display shifted Swan bands. Hence, they belong to the peculiar 

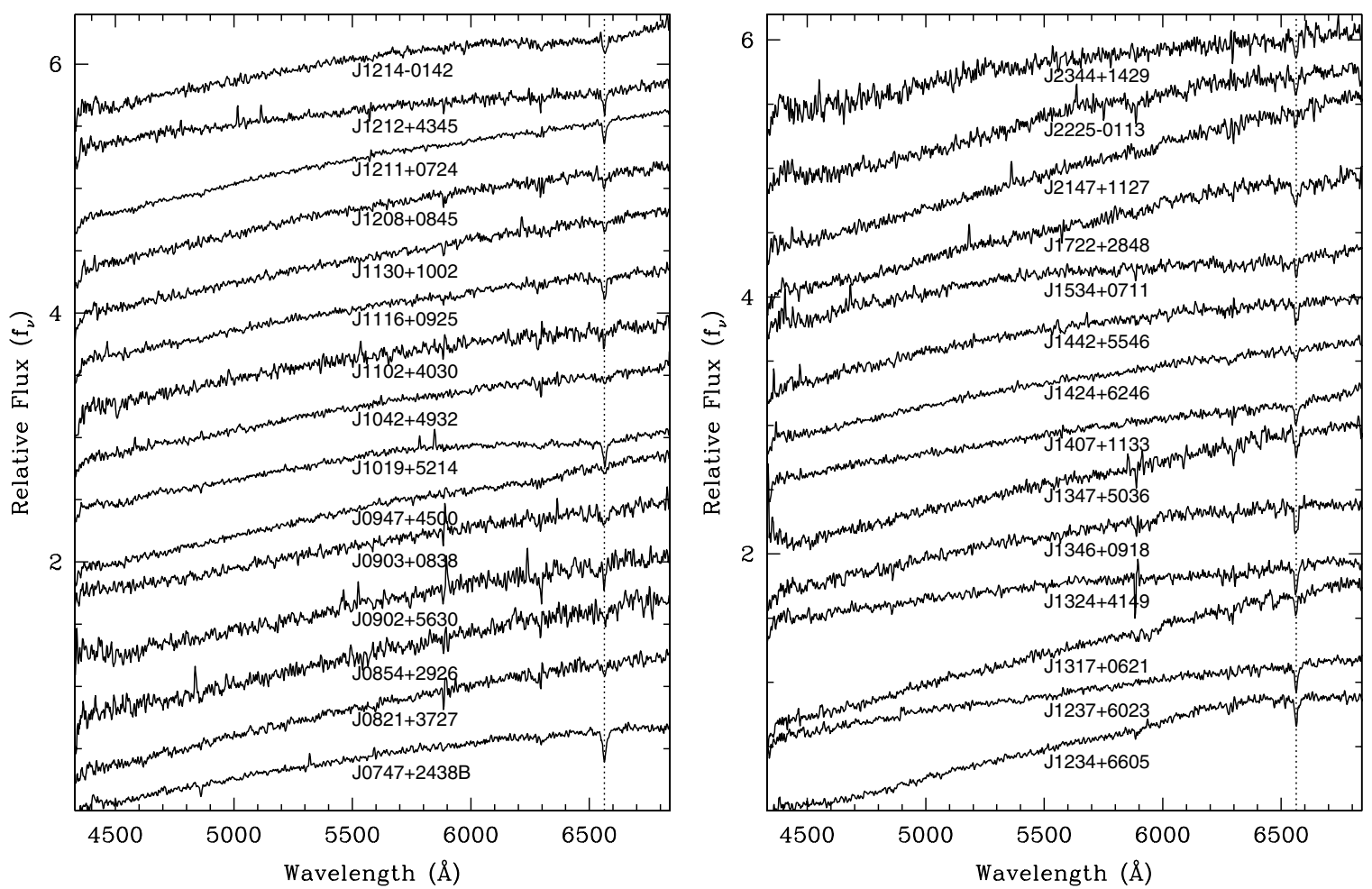

Figure 1. Optical spectra of the DA WDs observed at the HET. The spectra are normalized at $5700 \AA$ and are shifted vertically from each other by 0.4 units. The dotted line marks $\mathrm{H} \alpha$.
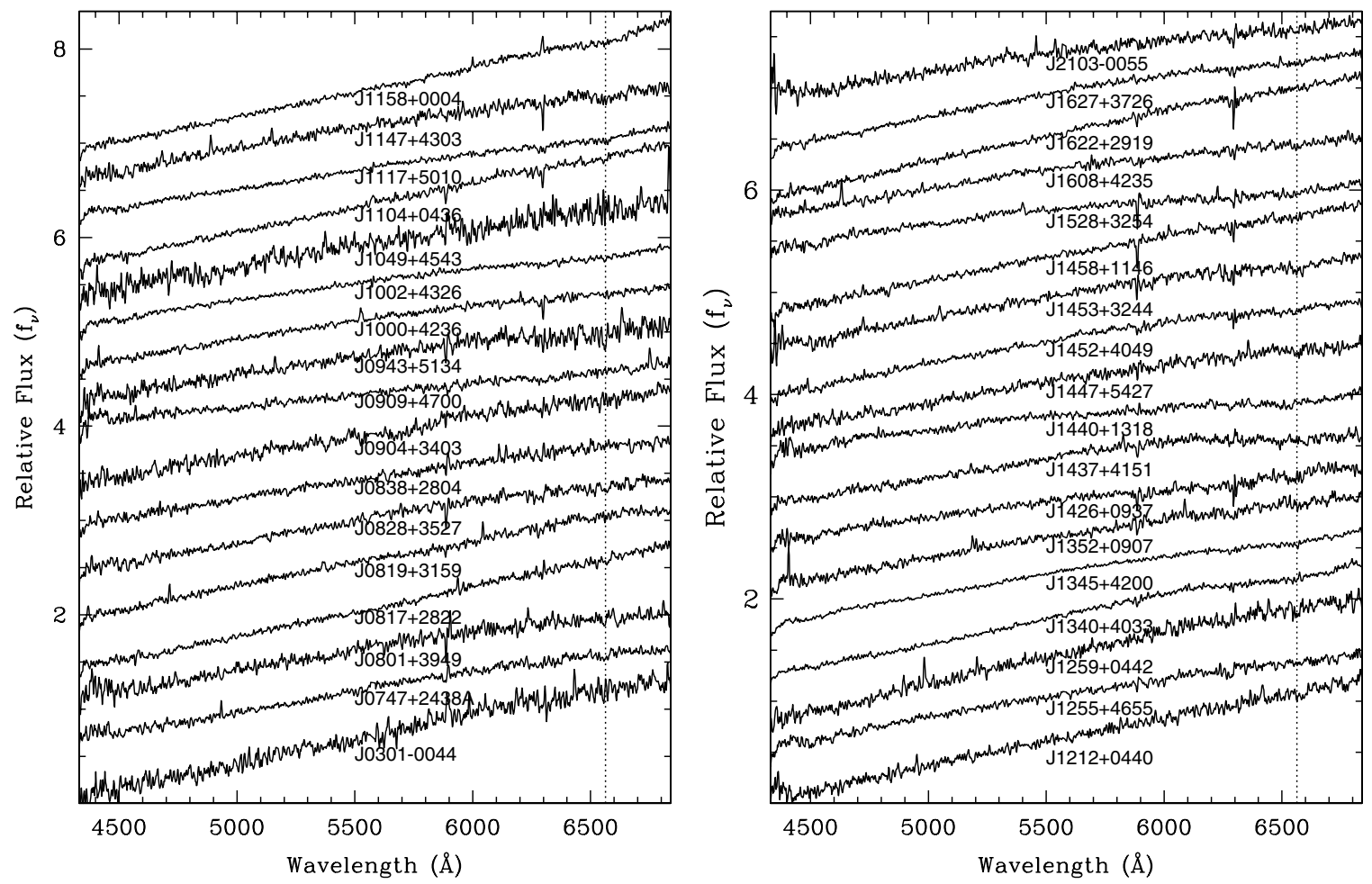

Figure 2. Optical spectra of the DC WDs observed at the HET.

DQ population (DQpec; Schmidt et al. 1995). Excluding the WD + dM pairs, J1247+0646 is the reddest WD in our sample with a $g-i$ color of 1.64 mag. The strong molecular absorption features in the blue causes this star to appear relatively red compared with all other DQ, DC, and DZ WDs.

The optical spectra of the stars presented in Figure 5 are dominated by M dwarfs, but the flux excess in the blue and the presence of strong $\mathrm{H} \beta$ and $\mathrm{H} \alpha$ absorption lines indicate DA WD companions. In addition, the $u$ - and $g$-band photometry for these stars is significantly brighter than expected from single $\mathrm{M}$ dwarf stars. These two stars are best explained as DA WD + dM pairs.

The coordinates, optical and near-infrared photometry, and spectroscopic classifications for our sample are given in Table 1. 


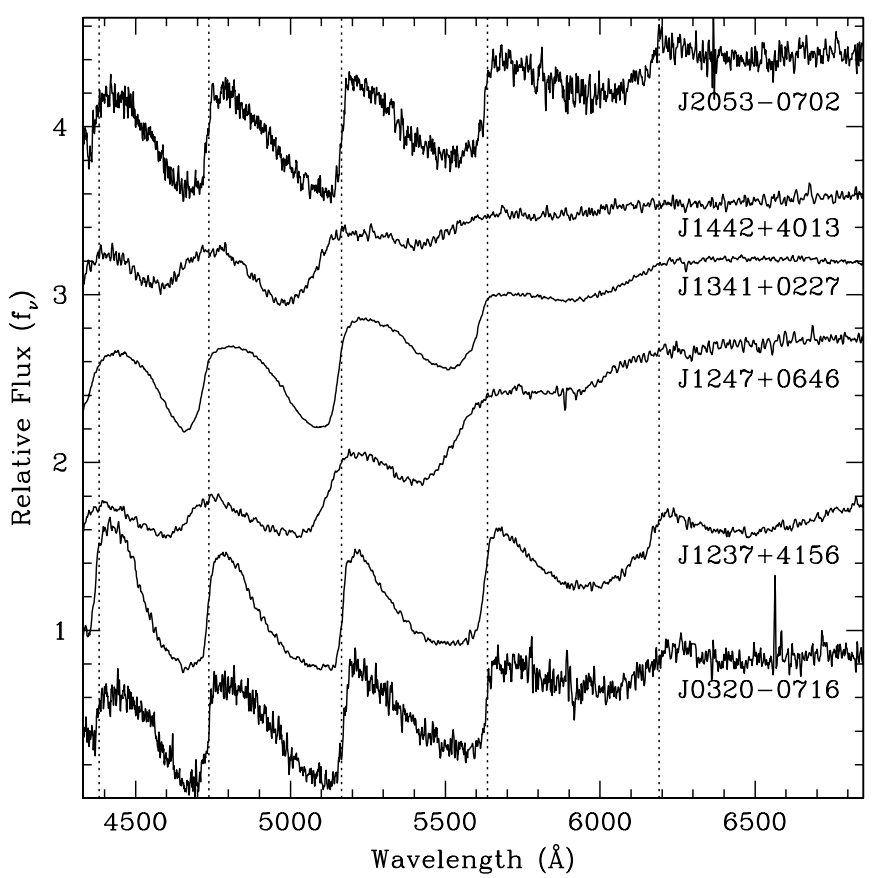

Figure 3. Optical spectra of the normal and peculiar DQ WDs in our sample. Dotted lines mark the expected locations of $\mathrm{C}_{2}$ bandheads. The spectra for J0320-0716 and J2053-0702 are from the SDSS.

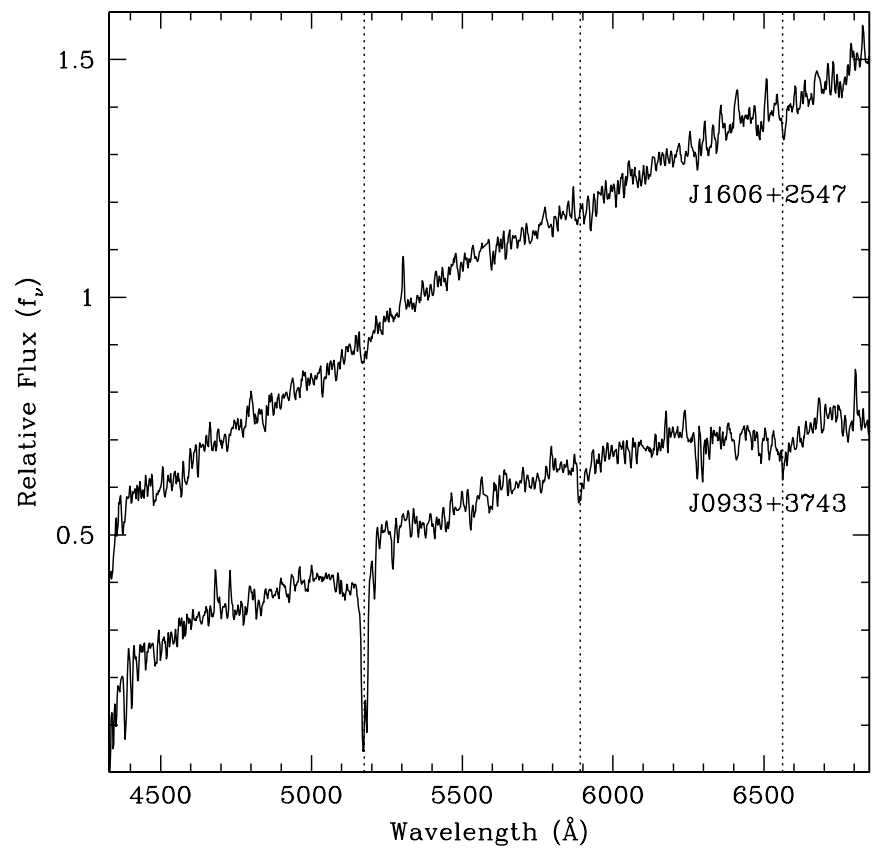

Figure 4. HET spectra of the DZA WDs. The dotted lines mark the positions of $\mathrm{Mg}, \mathrm{Na}$, and $\mathrm{H}$ absorption lines.

Positions are those from the SDSS astrometric pipeline (Pier et al. 2003). The photometric calibration is based on the SDSS standard star system (Smith et al. 2002) tied to the survey data with the Photometric Telescope (Hogg et al. 2001). The SDSS photometry is in the $\mathrm{AB}$ system. We use the corrections given in Eisenstein et al. (2006) for the $u-, i-$, and $z$-band photometry, and Table 1 includes these corrections. Even though this table includes 156 stars, optical spectroscopy (this paper; Kilic et al. 2006a, and the literature) and near-infrared photometry are available only for 140 and 126 stars, respectively. $J-, H$-, and $K$-band photometry is missing for 30,32 , and 38 stars in Table 1 ,

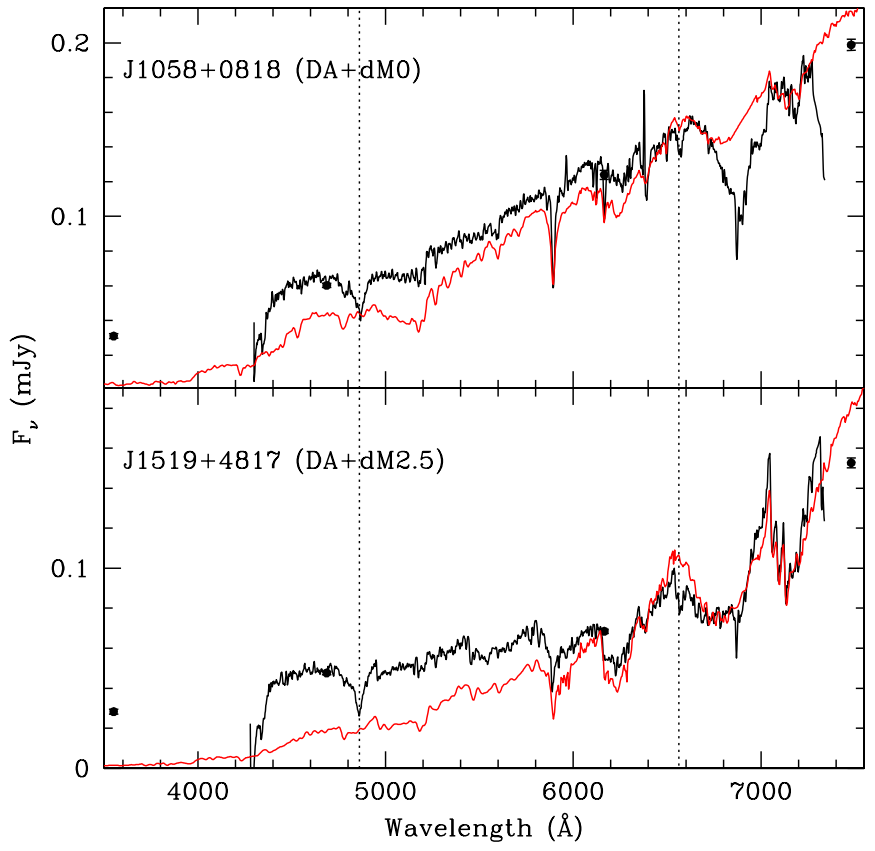

Figure 5. HET spectra of the WD+dM binaries. The observed spectra are normalized to match the SDSS $r$-band photometry. Red lines show the predicted contribution from dM companions based on Pickles (1998) templates. The blue excess from WD companions is evident in our HET spectra and the SDSS $u$ and $g$-band photometry. The dotted lines mark $\mathrm{H} \beta$ and $\mathrm{H} \alpha$.

(A color version of this figure is available in the online journal.)

respectively. The 30 stars with optical-only data (without $J$-band photometry) are not included in our model atmosphere analysis (Section 3.3). Out of the 140 stars with optical spectroscopy, 43 $(31 \%)$ are DA, $84(60 \%)$ are DC, $6(4 \%)$ are DQ, $5(4 \%)$ are $\mathrm{DZ}$, and $2(1 \%)$ are WD $+\mathrm{dM}$ pairs.

\subsection{Reduced Proper Motion Diagram}

Figure 6 shows the reduced proper motion diagram for the SDSS DR3 area including spectroscopic classifications from the SDSS and our observations. The SDSS observes mostly hotter WDs with $g-i<0.3 \mathrm{mag}$, whereas we focus on WDs with $g-i \geqslant 0.6 \mathrm{mag}$. Both of these selection effects are evident in this diagram. Compared with a few percent contamination rate from subdwarfs found by Kilic et al. (2006a), our sample has a higher rate of contamination from subdwarfs. Out of the 98 newly observed targets, 26 are subdwarfs. Table 2 presents the coordinates, optical photometry, and proper motions for these stars. Figure 7 presents their spectra. They all show $\mathrm{MgH}, \mathrm{Na}$, and several other metal lines. ${ }^{11}$ With a tangential velocity cutoff of $20 \mathrm{~km} \mathrm{~s}^{-1}$, many of our targets fall in the overlap region between the WD sequence and subdwarfs in the reduced proper motion diagram. Hence, the higher subdwarf contamination rate in this study is not surprising.

The cool WD selection works well for $V_{\tan } \geqslant 30 \mathrm{~km} \mathrm{~s}^{-1}$. Only one of the 75 cool WD candidates (J09194811+4356216, Figure 7) in the Harris et al. (2006) $V_{\tan } \geqslant 30 \mathrm{~km} \mathrm{~s}^{-1}$ and $M_{\mathrm{bol}}>14.6 \mathrm{mag}$ sample is actually a subdwarf, which corresponds to a contamination rate of $1.3 \%$. On the other hand, 4 out of $86 \operatorname{targets}(4.7 \%)$ with $V_{\tan } \geqslant 25 \mathrm{~km} \mathrm{~s}^{-1}$ and 30 out of 122 targets $(24.6 \%)$ with $V_{\tan } \geqslant 20 \mathrm{~km} \mathrm{~s}^{-1}$ are subdwarfs. Hence, increasing the lower tangential velocity limit from 20 to

11 The absorption feature at $6800 \AA$ is the atmospheric $B$ band. 
Table 1

Optical and Near-infrared Photometry of Cool White Dwarfs

\begin{tabular}{|c|c|c|c|c|c|c|c|c|c|c|}
\hline Name (SDSS J) & $u$ & $g$ & $r$ & $i$ & $z$ & $J$ & $H$ & $K$ & Type & Source \\
\hline $00: 03: 16.69-01: 11: 17.9$ & 20.53 & 19.32 & 18.79 & 18.56 & 18.52 & $17.63 \pm 0.04$ & $17.31 \pm 0.04$ & $17.35 \pm 0.05$ & DA & 1 \\
\hline $00: 33: 00.80+14: 51: 09.8$ & 19.27 & 18.56 & 18.13 & 17.99 & 18.01 & $17.39 \pm 0.05$ & $17.45 \pm 0.08$ & $\cdots$ & $\ldots$ & $\ldots$ \\
\hline $00: 45: 21.88+14: 20: 45.3$ & 20.64 & 19.20 & 18.45 & 18.20 & 18.10 & $17.24 \pm 0.04$ & $16.99 \pm 0.04$ & $16.89 \pm 0.04$ & DZA & 1 \\
\hline 01:02:59.98+14:01:08.1 & 21.21 & 19.48 & 18.69 & 18.41 & 18.30 & $17.39 \pm 0.04$ & $17.05 \pm 0.04$ & $17.05 \pm 0.06$ & DC & 1 \\
\hline $01: 46: 29.01+14: 04: 38.2$ & 21.21 & 19.99 & 19.37 & 19.24 & 19.71 & $19.56 \pm 0.05$ & $20.07 \pm 0.12$ & 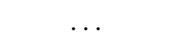 & DC & 3 \\
\hline $01: 57: 43.25+13: 35: 58.2$ & 20.56 & 19.35 & 18.65 & 18.47 & 18.41 & $17.75 \pm 0.04$ & $17.53 \pm 0.04$ & $17.49 \pm 0.06$ & DC & 1 \\
\hline $02: 12: 06.36-00: 40: 05.8$ & 19.80 & 18.96 & 18.59 & 18.41 & 18.39 & $17.60 \pm 0.04$ & $17.27 \pm 0.04$ & $17.42 \pm 0.05$ & $\ldots$ & $\ldots$ \\
\hline 02:50:05.81-09:10:02.8 & 20.02 & 18.96 & 18.45 & 18.27 & 18.21 & $17.41 \pm 0.04$ & $17.14 \pm 0.04$ & $17.03 \pm 0.06$ & DA & 1 \\
\hline $02: 56: 41.62-07: 00: 33.8$ & 20.74 & 19.00 & 18.13 & 17.79 & 17.69 & $16.71 \pm 0.05$ & $16.62 \pm 0.05$ & $16.48 \pm 0.06$ & DC & 1 \\
\hline $03: 01: 44.09-00: 44: 39.5$ & 22.23 & 20.43 & 19.38 & 18.99 & 18.92 & $17.96 \pm 0.04$ & $17.73 \pm 0.04$ & $17.68 \pm 0.08$ & DC & 2 \\
\hline 03:07:13.91-07:15:06.2 & 18.65 & 17.65 & 17.18 & 17.01 & 16.98 & $16.20 \pm 0.04$ & $15.95 \pm 0.04$ & $15.87 \pm 0.05$ & DA & 4 \\
\hline $03: 09: 24.87+00: 25: 25.3$ & 19.15 & 18.19 & 17.72 & 17.53 & 17.50 & $16.64 \pm 0.04$ & $16.54 \pm 0.04$ & $16.87 \pm 0.04$ & DC & 1 \\
\hline $03: 10: 49.53-01: 10: 35.3$ & 22.49 & 20.95 & 20.20 & 19.89 & 19.97 & $18.94 \pm 0.02$ & $18.73 \pm 0.02$ & $18.58 \pm 0.02$ & DC & 3 \\
\hline $03: 14: 49.81-01: 05: 19.3$ & 19.53 & 18.59 & 18.14 & 17.98 & 17.97 & $17.08 \pm 0.04$ & $16.82 \pm 0.04$ & $16.85 \pm 0.06$ & DA & 1 \\
\hline $03: 20: 54.11-07: 16: 25.4$ & 19.93 & 19.75 & 19.27 & 19.21 & 19.19 & $18.80 \pm 0.04$ & $18.69 \pm 0.05$ & $18.62 \pm 0.06$ & DQ & 3 \\
\hline 03:30:54.88+00:37:16.5 & 20.72 & 19.79 & 19.32 & 19.16 & 19.10 & $18.35 \pm 0.04$ & $18.16 \pm 0.05$ & $18.09 \pm 0.08$ & DA & 1 \\
\hline $04: 06: 47.32-06: 44: 36.9$ & 18.83 & 18.02 & 17.58 & 17.48 & 17.40 & $16.59 \pm 0.05$ & $16.44 \pm 0.04$ & $16.27 \pm 0.05$ & DA & 1 \\
\hline $07: 47: 21.56+24: 38: 47.7$ & 21.09 & 19.27 & 18.54 & 18.26 & 18.17 & $17.16 \pm 0.04$ & $16.99 \pm 0.04$ & $16.85 \pm 0.04$ & DC & 2 \\
\hline $07: 47: 23.50+24: 38: 23.7$ & 19.34 & 18.36 & 17.92 & 17.75 & 17.72 & $16.78 \pm 0.04$ & $16.58 \pm 0.04$ & $16.53 \pm 0.05$ & DA & 2 \\
\hline $07: 53: 13.28+42: 30: 01.6$ & 19.97 & 18.09 & 17.19 & 16.87 & 16.75 & $15.69 \pm 0.04$ & $15.49 \pm 0.04$ & $15.47 \pm 0.04$ & DC & 1 \\
\hline $08: 01: 32.83+39: 49: 25.9$ & 21.88 & 20.17 & 19.37 & 19.09 & 18.96 & & & & DC & 2 \\
\hline $08: 04: 40.63+22: 39: 48.7$ & 19.73 & 18.30 & 17.59 & 17.39 & 17.33 & $16.71 \pm 0.04$ & $16.92 \pm 0.04$ & $17.29 \pm 0.06$ & $\mathrm{DZ}$ & 2 \\
\hline $08: 17: 45.33+24: 51: 05.5$ & 20.59 & 19.44 & 18.91 & 18.74 & 18.62 & $17.93 \pm 0.04$ & $17.76 \pm 0.04$ & $17.71 \pm 0.09$ & $\ldots$ & $\ldots$ \\
\hline $08: 17: 51.52+28: 22: 03.1$ & 21.59 & 19.49 & 18.61 & 18.30 & 18.22 & $17.33 \pm 0.04$ & $17.01 \pm 0.04$ & $16.91 \pm 0.09$ & DC & 2 \\
\hline $08: 19: 24.32+31: 59: 56.8$ & 21.65 & 19.75 & 18.90 & 18.59 & 18.43 & $17.47 \pm 0.04$ & $17.32 \pm 0.04$ & $17.21 \pm 0.09$ & DC & 2 \\
\hline $08: 21: 08.18+37: 27: 38.3$ & 20.68 & 19.14 & 18.43 & 18.15 & 18.04 & $17.25 \pm 0.04$ & $17.00 \pm 0.04$ & $16.85 \pm 0.05$ & DA & 2 \\
\hline $08: 25: 00.61+28: 41: 49.9$ & 20.14 & 18.98 & 18.44 & 18.29 & 18.18 & $17.38 \pm 0.04$ & $17.12 \pm 0.04$ & $17.03 \pm 0.04$ & - & \\
\hline $08: 25: 19.70+50: 49: 20.1$ & 21.09 & 19.34 & 18.43 & 18.09 & 18.00 & $17.08 \pm 0.04$ & $16.83 \pm 0.04$ & $16.74 \pm 0.04$ & DC & 1 \\
\hline $08: 28: 42.31+35: 27: 29.5$ & 21.43 & 19.85 & 19.05 & 18.73 & 18.71 & $\ldots$ & $\ldots$ & & DC & 2 \\
\hline $08: 36: 41.56+45: 56: 58.7$ & 21.64 & 20.01 & 19.14 & 18.87 & 18.73 & $17.90 \pm 0.04$ & $17.70 \pm 0.04$ & $17.59 \pm 0.05$ & DC & 1 \\
\hline $08: 38: 31.82+28: 04: 59.7$ & 20.55 & 19.14 & 18.49 & 18.29 & 18.21 & $17.33 \pm 0.04$ & $17.07 \pm 0.04$ & $17.04 \pm 0.05$ & DC & 2 \\
\hline $08: 53: 45.93+41: 18: 50.1$ & 20.38 & 19.33 & 18.75 & 18.59 & 18.60 & $17.83 \pm 0.04$ & $17.70 \pm 0.05$ & $17.67 \pm 0.06$ & $\ldots$ & $\ldots$ \\
\hline $08: 54: 54.45+29: 26: 41.8$ & 20.80 & 19.33 & 18.63 & 18.48 & 18.36 & & & & DA & 2 \\
\hline $08: 54: 43.33+35: 03: 52.7$ & 23.57 & 20.53 & 19.39 & 19.09 & 18.95 & $18.44 \pm 0.04$ & $18.23 \pm 0.04$ & $17.98 \pm 0.04$ & DC & 5 \\
\hline 08:55:49.89+37:00:16.7 & 19.01 & 17.97 & 17.47 & 17.29 & 17.27 & $16.37 \pm 0.05$ & $16.17 \pm 0.04$ & $16.08 \pm 0.05$ & $\ldots$ & $\ldots$ \\
\hline $09: 02: 44.02+56: 30: 32.7$ & 20.84 & 19.56 & 18.88 & 18.70 & 18.52 & $\ldots$ & $\ldots$ & $\ldots$ & DA & 2 \\
\hline 09:03:04.38+08:38:15.6 & 20.06 & 18.85 & 18.27 & 18.08 & 17.99 & $\ldots$ & $\ldots$ & $\ldots$ & $\mathrm{DA}$ & 2 \\
\hline 09:04:06.89+34:03:53.1 & 20.01 & 18.73 & 18.10 & 17.87 & 17.85 & $17.23 \pm 0.04$ & $16.93 \pm 0.05$ & $16.85 \pm 0.06$ & DC & 2 \\
\hline $09: 09: 14.56+47: 00: 17.5$ & 20.64 & 19.29 & 18.74 & 18.50 & 18.42 & $18.11 \pm 0.04$ & $18.62 \pm 0.07$ & $19.10 \pm 0.10$ & DC & 2 \\
\hline $09: 33: 45.58+37: 43: 49.8$ & 20.71 & 19.17 & 18.59 & 18.42 & 18.36 & $17.63 \pm 0.04$ & $17.37 \pm 0.04$ & $17.26 \pm 0.04$ & DZA & 2 \\
\hline $09: 42: 44.96+44: 37: 43.1$ & 21.37 & 19.47 & 18.58 & 18.22 & 18.05 & $17.15 \pm 0.04$ & $16.97 \pm 0.04$ & $16.86 \pm 0.04$ & DC & 1 \\
\hline $09: 43: 16.62+51: 34: 40.9$ & 20.52 & 18.89 & 18.13 & 17.84 & 17.75 & $16.72 \pm 0.05$ & $16.61 \pm 0.06$ & $16.53 \pm 0.06$ & $\mathrm{DC}$ & 2 \\
\hline $09: 47: 23.00+44: 59: 48.7$ & 20.67 & 19.44 & 18.84 & 18.95 & 19.45 & $19.69 \pm 0.07$ & $20.34 \pm 0.06$ & $20.96 \pm 0.12$ & DC & 5 \\
\hline 09:47:24.47+45:00:01.9 & 21.27 & 19.52 & 18.77 & 18.53 & 18.32 & $17.43 \pm 0.04$ & $17.24 \pm 0.04$ & $17.11 \pm 0.04$ & DA & 5 \\
\hline $10: 00: 29.47+42: 36: 31.2$ & 21.70 & 19.58 & 18.79 & 18.46 & 18.39 & $17.47 \pm 0.04$ & $17.23 \pm 0.04$ & $17.13 \pm 0.05$ & DC & 2 \\
\hline $10: 01: 03.42+39: 03: 40.5$ & 21.36 & 20.05 & 19.60 & 20.02 & 20.61 & $20.65 \pm 0.06$ & $21.05 \pm 0.07$ & $\ldots$ & DC & 5 \\
\hline $10: 01: 19.48+46: 56: 50.6$ & 21.34 & 19.27 & 18.22 & 17.90 & 17.82 & $16.79 \pm 0.04$ & $16.69 \pm 0.06$ & $16.99 \pm 0.06$ & DC & 1 \\
\hline $10: 02: 04.11+43: 26: 45.7$ & 20.40 & 18.56 & 17.74 & 17.45 & 17.30 & & & & DC & 2 \\
\hline 10:02:25.85+61:08:58.1 & 21.70 & 19.40 & 18.42 & 18.06 & 17.87 & $16.85 \pm 0.06$ & $16.69 \pm 0.07$ & $16.72 \pm 0.05$ & DC & 1 \\
\hline $10: 19: 59.36+52: 14: 08.4$ & 19.83 & 18.51 & 17.95 & 17.73 & 17.67 & $\ldots$ & $\ldots$ & & DA & 2 \\
\hline $10: 42: 44.79+49: 32: 47.0$ & 20.62 & 19.17 & 18.59 & 18.40 & 18.31 & $17.51 \pm 0.04$ & $17.32 \pm 0.04$ & $17.32 \pm 0.05$ & $\mathrm{DC}$ & 2 \\
\hline $10: 49: 39.97+45: 43: 57.5$ & 21.97 & 20.04 & 19.18 & 18.87 & 18.74 & $\ldots$ & $\ldots$ & $\ldots$ & DC & 2 \\
\hline $10: 58: 35.49+08: 18: 28.6$ & 20.17 & 19.45 & 18.67 & 18.15 & 17.81 & $\ldots$ & $\ldots$ & & WD+dM & 2 \\
\hline $11: 02: 13.70+67: 07: 52.6$ & 21.37 & 19.62 & 18.95 & 18.68 & 18.65 & $17.76 \pm 0.04$ & $17.49 \pm 0.04$ & $17.41 \pm 0.05$ & DC & 1 \\
\hline $11: 02: 29.26+40: 30: 04.8$ & 20.44 & 18.90 & 18.24 & 18.00 & 17.87 & $17.09 \pm 0.04$ & $16.78 \pm 0.04$ & $16.67 \pm 0.04$ & DA & 2 \\
\hline $11: 04: 00.98+04: 36: 16.8$ & 22.06 & 19.90 & 18.96 & 18.54 & 18.46 & $17.42 \pm 0.05$ & $17.31 \pm 0.06$ & $17.26 \pm 0.06$ & DC & 2 \\
\hline $11: 07: 31.38+48: 55: 23.0$ & 21.50 & 19.49 & 18.54 & 18.23 & 18.11 & $17.05 \pm 0.05$ & $16.95 \pm 0.07$ & $16.86 \pm 0.07$ & DC & 1 \\
\hline 11:16:08.81+09:25:32.6 & 20.26 & 19.03 & 18.47 & 18.25 & 18.22 & $17.31 \pm 0.04$ & $17.09 \pm 0.04$ & $16.98 \pm 0.05$ & DA & 2 \\
\hline $11: 17: 08.63+50: 10: 33.9$ & 21.17 & 19.34 & 18.57 & 18.30 & 18.16 & $17.24 \pm 0.04$ & $17.07 \pm 0.04$ & $16.97 \pm 0.05$ & DC & 2 \\
\hline $11: 19: 40.62-01: 07: 55.1$ & 21.99 & 19.95 & 19.06 & 18.80 & 18.65 & $17.76 \pm 0.04$ & $17.55 \pm 0.04$ & $17.41 \pm 0.05$ & DC & 1 \\
\hline 11:30:50.44+10:02:59.3 & 20.41 & 18.89 & 18.23 & 17.98 & 17.89 & $17.10 \pm 0.04$ & $16.85 \pm 0.05$ & $16.77 \pm 0.06$ & DA & 2 \\
\hline 11:47:09.81+43:03:06.4 & 20.60 & 19.09 & 18.33 & 18.04 & 17.95 & $17.04 \pm 0.04$ & $16.84 \pm 0.04$ & $16.72 \pm 0.04$ & DC & 2 \\
\hline $11: 51: 16.42+12: 53: 45.6$ & 20.72 & 19.38 & 18.80 & 18.57 & 18.48 & $17.64 \pm 0.04$ & $17.41 \pm 0.04$ & $17.45 \pm 0.05$ & DC & 6 \\
\hline $11: 58: 14.52+00: 04: 58.3$ & 20.86 & 18.89 & 17.85 & 17.54 & 17.34 & $16.36 \pm 0.04$ & $16.31 \pm 0.05$ & $16.18 \pm 0.05$ & DC & 2 \\
\hline $12: 02: 00.48-03: 13: 47.4$ & 22.34 & 19.97 & 19.08 & 18.77 & 18.68 & $17.61 \pm 0.05$ & $17.55 \pm 0.08$ & $17.51 \pm 0.09$ & DC & 1 \\
\hline $12: 04: 39.54+62: 22: 16.4$ & 20.91 & 19.25 & 18.43 & 18.14 & 18.06 & $17.07 \pm 0.04$ & $16.86 \pm 0.04$ & $16.80 \pm 0.04$ & $\mathrm{DC}$ & 1 \\
\hline
\end{tabular}


Table 1

(Continued)

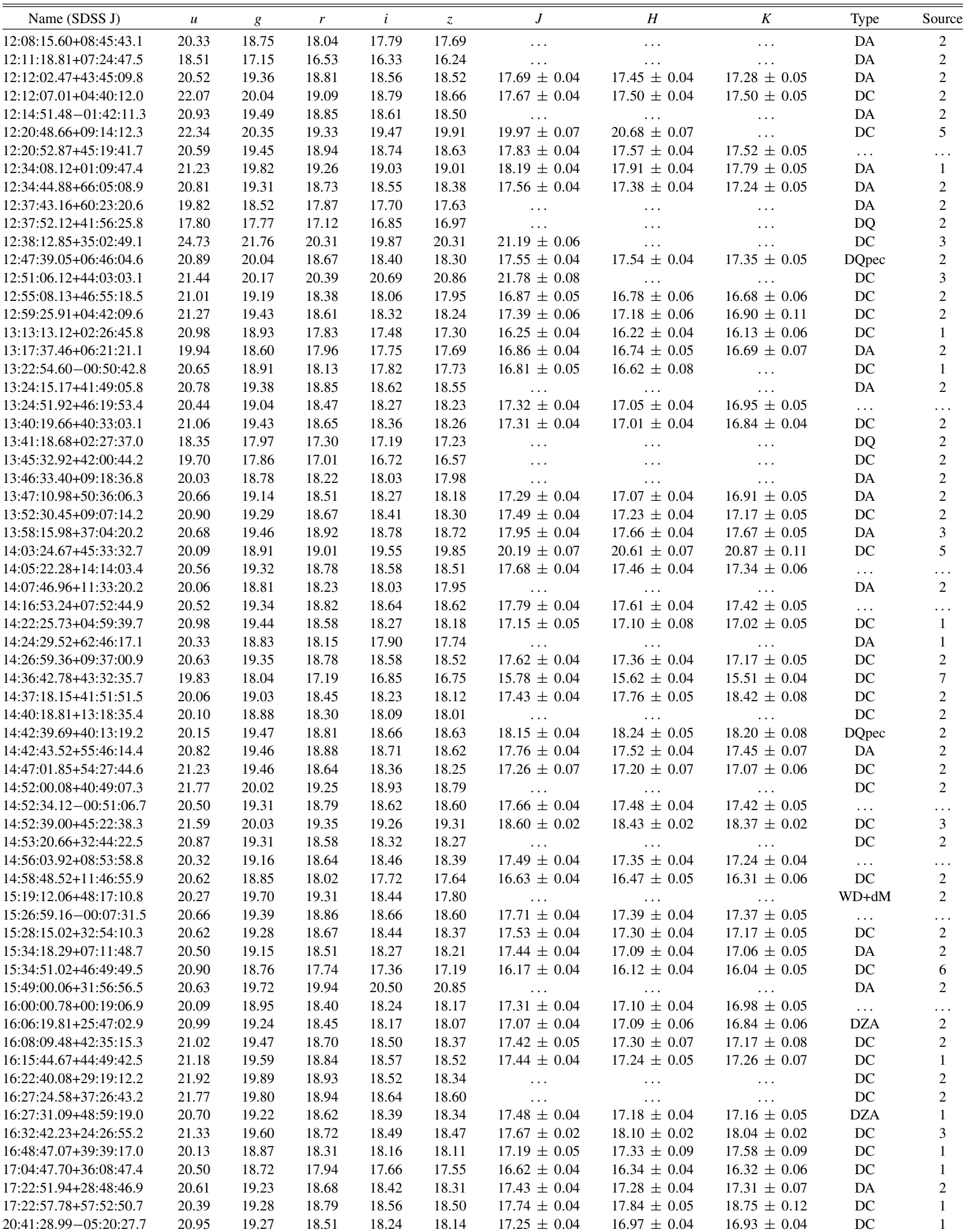


Table 1

(Continued)

\begin{tabular}{|c|c|c|c|c|c|c|c|c|c|c|}
\hline Name (SDSS J) & $u$ & $g$ & $r$ & $i$ & $z$ & $J$ & $H$ & $K$ & Type & Source \\
\hline $20: 42: 59.23+00: 31: 56.6$ & 21.67 & 19.95 & 19.05 & 18.73 & 18.61 & $17.65 \pm 0.04$ & $17.45 \pm 0.04$ & $17.36 \pm 0.05$ & DC & 1 \\
\hline $20: 45: 06.97+00: 37: 34.4$ & 20.45 & 19.77 & 19.42 & 19.26 & 19.21 & $18.43 \pm 0.04$ & $18.23 \pm 0.05$ & $18.22 \pm 0.08$ & DA & 1 \\
\hline $20: 45: 57.53-07: 10: 03.5$ & 21.00 & 19.33 & 18.60 & 18.34 & 18.18 & $17.32 \pm 0.04$ & $17.10 \pm 0.04$ & $17.03 \pm 0.04$ & DC & 1 \\
\hline $21: 03: 36.68-00: 55: 45.2$ & 21.65 & 20.06 & 19.29 & 19.02 & 18.89 & $\ldots$ & $\ldots$ & $\ldots$ & DC & 2 \\
\hline $21: 16: 40.30-07: 24: 52.7$ & 20.25 & 18.43 & 17.59 & 17.26 & 17.13 & $16.16 \pm 0.04$ & $16.02 \pm 0.05$ & $15.90 \pm 0.05$ & DC & 1 \\
\hline $21: 18: 05.21-07: 37: 29.1$ & 23.38 & 20.70 & 19.48 & 19.01 & 18.76 & $17.90 \pm 0.04$ & $17.82 \pm 0.04$ & $17.81 \pm 0.05$ & DC & 1 \\
\hline $21: 51: 53.79-07: 31: 31.0$ & 19.85 & 18.85 & 18.36 & 18.19 & 18.14 & $17.32 \pm 0.04$ & $16.96 \pm 0.04$ & $16.89 \pm 0.05$ & $\ldots$ & .. \\
\hline 22:04:14.16-01:09:31.2 & 22.16 & 20.21 & 19.29 & 18.99 & 18.83 & $18.01 \pm 0.04$ & $17.68 \pm 0.04$ & $17.70 \pm 0.05$ & $\mathrm{DC}$ & 1 \\
\hline $22: 25: 43.50-01: 13: 59.6$ & 21.47 & 19.90 & 19.12 & 18.89 & 18.75 & & & $\ldots$ & DA & 2 \\
\hline $22: 39: 54.07+00: 18: 49.2$ & 24.21 & 21.02 & 19.93 & 19.59 & 19.41 & $18.40 \pm 0.02$ & $18.25 \pm 0.02$ & $18.48 \pm 0.27$ & DC & 3 \\
\hline $22: 39: 54.12+00: 18: 47.3$ & 21.51 & 20.16 & 19.53 & 19.47 & 20.09 & $19.73 \pm 0.06$ & $19.99 \pm 0.08$ & & $\mathrm{DC}$ & 3 \\
\hline $22: 42: 06.19+00: 48: 22.8$ & 22.11 & 19.63 & 18.65 & 18.28 & 18.16 & $18.06 \pm 0.04$ & $18.72 \pm 0.07$ & $19.16 \pm 0.10$ & DC & 1 \\
\hline $23: 30: 55.20+00: 28: 52.3$ & 21.85 & 19.88 & 18.95 & 18.66 & 18.53 & $17.63 \pm 0.04$ & $17.36 \pm 0.04$ & $17.32 \pm 0.04$ & DC & 1 \\
\hline $23: 42: 45.75-10: 01: 21.4$ & 20.45 & 18.95 & 18.21 & 17.94 & 17.89 & $17.08 \pm 0.06$ & $16.90 \pm 0.06$ & $16.79 \pm 0.07$ & $\mathrm{DA}$ & 1 \\
\hline $23: 44: 05.54-14: 29: 23.5$ & 21.28 & 19.79 & 19.19 & 18.94 & 18.89 & $\ldots$ & $\ldots$ & $\ldots$ & DA & 2 \\
\hline $23: 50: 42.52-08: 46: 18.9$ & 20.22 & 19.17 & 18.61 & 18.38 & 18.31 & $17.52 \pm 0.04$ & $17.27 \pm 0.04$ & $17.19 \pm 0.05$ & $\mathrm{DA}$ & 1 \\
\hline
\end{tabular}

Notes. The last column in the table refers to the source of the optical spectroscopic observations: (1) Kilic et al. 2006; (2) This paper; (3) SDSS; (4) Oppenheimer et al. 2001; (5) Gates et al. 2004; (6) Oswalt et al. 1996; (7) Hintzen 1986; (8) Carollo et al. 2006; (9) Vennes \& Kawka 2003.

Table 2

Spectroscopically Identified Subdwarf Stars

\begin{tabular}{|c|c|c|c|c|c|c|c|c|}
\hline Name (SDSS J) & $\begin{array}{c}u \\
(\mathrm{mag})\end{array}$ & $\begin{array}{c}g \\
(\mathrm{mag})\end{array}$ & $\begin{array}{c}r \\
(\mathrm{mag})\end{array}$ & $\begin{array}{c}i \\
(\mathrm{mag})\end{array}$ & $\begin{array}{c}z \\
(\mathrm{mag})\end{array}$ & $\begin{array}{c}\mu_{\text {R.A. }} \\
\left(\mathrm{mas} \mathrm{yr}^{-1}\right)\end{array}$ & $\begin{array}{c}\mu_{\text {Decl. }} \\
\left(\mathrm{mas} \mathrm{yr}^{-1}\right)\end{array}$ & $\overline{\text { Epochs }}$ \\
\hline $09: 19: 48.11+43: 56: 21.6$ & 21.90 & 19.42 & 18.45 & 17.94 & 17.60 & -73 & -171 & 6 \\
\hline $10: 13: 29.64+51: 04: 12.8$ & 20.83 & 18.57 & 17.47 & 17.03 & 16.80 & -117 & -156 & 6 \\
\hline $10: 19: 57.78+62: 19: 48.1$ & 20.57 & 18.48 & 17.63 & 17.30 & 17.18 & -83 & -128 & 6 \\
\hline $10: 21: 36.30+38: 08: 39.8$ & 21.09 & 18.80 & 17.68 & 17.23 & 17.00 & 24 & -199 & 6 \\
\hline $10: 29: 22.43+02: 44: 53.3$ & 21.96 & 19.49 & 18.36 & 17.87 & 17.64 & -86 & -103 & 6 \\
\hline $11: 06: 10.50+11: 34: 24.2$ & 20.53 & 18.77 & 17.84 & 17.48 & 17.26 & -77 & -97 & 6 \\
\hline $11: 19: 58.69+43: 54: 54.8$ & 21.72 & 19.46 & 18.43 & 18.02 & 17.75 & -18 & -103 & 6 \\
\hline $12: 00: 18.05+11: 46: 48.4$ & 20.57 & 18.63 & 17.67 & 17.27 & 17.05 & -116 & -166 & 6 \\
\hline $12: 04: 50.43+05: 11: 54.1$ & 21.44 & 19.22 & 18.04 & 17.57 & 17.30 & -41 & -164 & 6 \\
\hline $12: 08: 51.72+43: 24: 10.4$ & 20.81 & 18.53 & 17.39 & 16.96 & 16.64 & -183 & 24 & 6 \\
\hline $12: 33: 30.40+10: 00: 31.8$ & 20.87 & 18.81 & 17.77 & 17.35 & 17.10 & -54 & -159 & 6 \\
\hline $12: 34: 13.46+02: 01: 39.2$ & 21.42 & 18.80 & 17.65 & 17.17 & 16.93 & -183 & -54 & 6 \\
\hline $12: 39: 07.85+47: 22: 16.5$ & 21.53 & 19.46 & 18.39 & 17.99 & 17.76 & -82 & -69 & 6 \\
\hline $12: 44: 25.95-01: 44: 25.2$ & 19.66 & 17.51 & 16.48 & 16.03 & 15.80 & -217 & -187 & 6 \\
\hline $13: 11: 06.30+51: 54: 45.0$ & 19.93 & 17.76 & 16.75 & 16.30 & 16.09 & -14 & -262 & 6 \\
\hline $13: 12: 29.65+41: 02: 20.5$ & 21.47 & 19.44 & 18.41 & 17.98 & 17.77 & -49 & -100 & 6 \\
\hline $13: 12: 43.88+59: 27: 10.1$ & 21.66 & 19.47 & 18.33 & 17.86 & 17.66 & 11 & -125 & 6 \\
\hline $13: 16: 33.66+02: 28: 17.9$ & 20.52 & 18.49 & 17.45 & 17.07 & 16.84 & -63 & -170 & 6 \\
\hline $13: 37: 15.76+01: 14: 56.4$ & 20.75 & 18.44 & 17.33 & 16.86 & 16.66 & -145 & -141 & 6 \\
\hline $13: 58: 18.78+03: 22: 59.5$ & 19.29 & 17.08 & 15.97 & 15.54 & 15.34 & -257 & -273 & 6 \\
\hline $14: 20: 28.35+07: 24: 54.5$ & 21.15 & 19.16 & 18.11 & 17.71 & 17.48 & -138 & -132 & 4 \\
\hline $14: 23: 24.99+12: 40: 38.1$ & 20.32 & 18.25 & 17.42 & 17.07 & 16.86 & -174 & 94 & 6 \\
\hline $14: 49: 29.72+34: 28: 43.2$ & 21.86 & 19.43 & 18.30 & 17.83 & 17.60 & -107 & -102 & 6 \\
\hline $14: 58: 44.11+00: 44: 03.6$ & 21.13 & 19.48 & 18.60 & 18.26 & 18.07 & 16 & 87 & 6 \\
\hline $15: 11: 45.76+03: 31: 16.2$ & 25.36 & 17.80 & 16.67 & 16.20 & 15.98 & -210 & -210 & 6 \\
\hline $16: 06: 44.68+48: 34: 51.6$ & 21.43 & 19.07 & 17.99 & 17.55 & 17.32 & -154 & 28 & 6 \\
\hline
\end{tabular}

Notes. The proper motion measurements are from Munn et al. (2004). The last column indicates the number of epochs an object is detected in the USNO-B + SDSS.

$30 \mathrm{~km} \mathrm{~s}^{-1}$ gets rid of most of the contamination from subdwarfs. The $V_{\tan }=30 \mathrm{~km} \mathrm{~s}^{-1}$ curve is the best choice for creating a clean sample of WDs without losing too many of them in the overlap region with subdwarfs. This selection can be used to create clean samples of cool WDs from the SDSS DR7 and SEGUE data and (depending on the astrometric accuracy) future surveys that use 


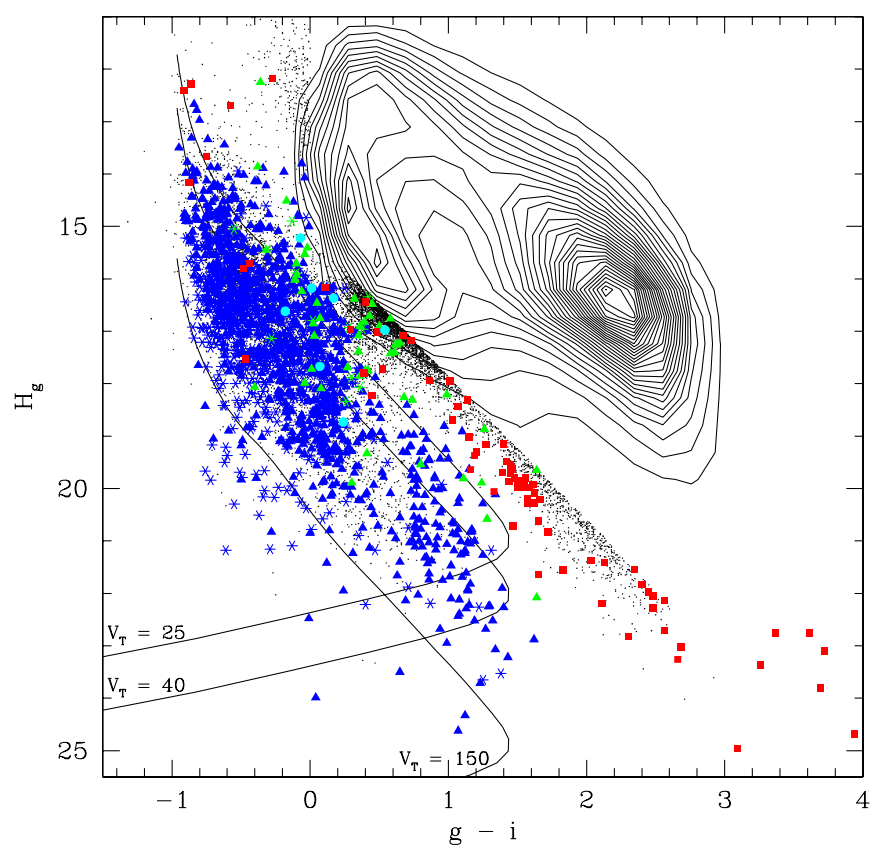

Figure 6. Reduced proper motion diagram for stars in the SDSS DR3. Individual stars are plotted only in the region of interest for WDs, the remaining stars are represented by the contours. WDs, WDs plus late-type star binaries, subdwarfs, and quasars are shown as blue triangles, green triangles, red squares, and cyan circles, respectively. WDs that do not meet our criteria for reliable proper motions (see Kilic et al. 2006a) are plotted as blue asterisks. WD cooling curves for different tangential velocities are shown as solid lines. The $V_{\mathrm{T}}=$ $25-40 \mathrm{~km} \mathrm{~s}^{-1}$ curves mark the expected location of disk WDs, whereas the $V_{\mathrm{T}}=150 \mathrm{~km} \mathrm{~s}^{-1}$ curve represents the halo WDs.

(A color version of this figure is available in the online journal.)

the SDSS filters (e.g., the SkyMapper, Pan-STARRS, and the Large Synoptic Survey Telescope (LSST)).

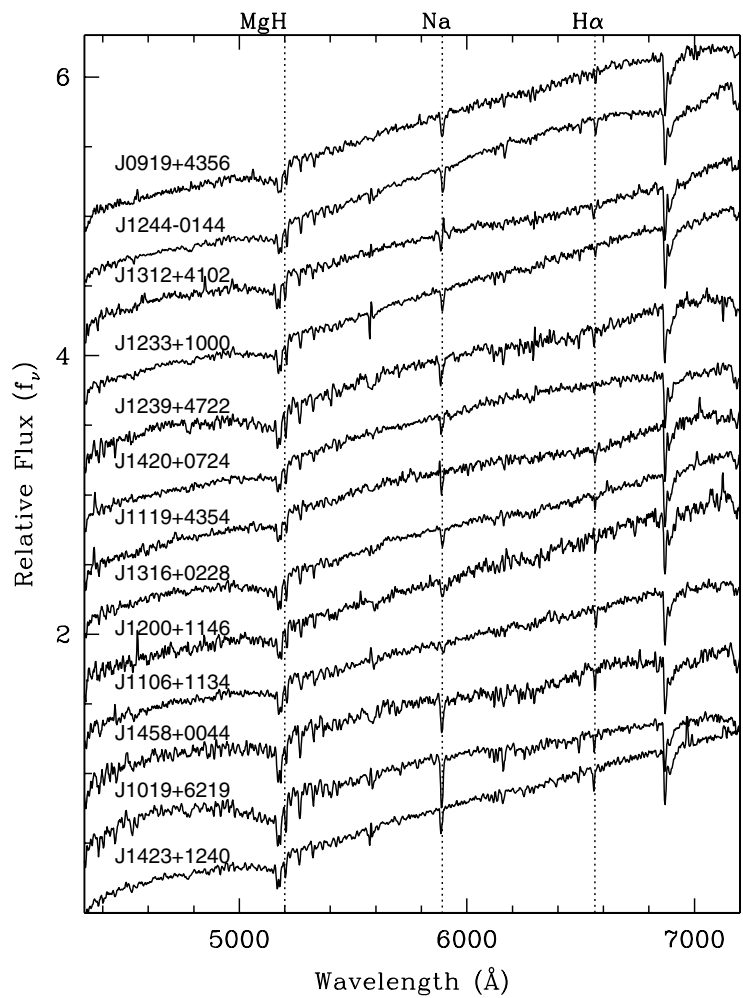

\subsection{Color-Color Diagrams}

Figure 8 shows the optical color-color diagrams for the cool WDs in Kilic et al. (2006a), Gates et al. (2004), Harris et al. (2008), and this paper. A comparison of the observed colors with the predicted colors of pure hydrogen and pure helium atmosphere WD models (see Section 3.3) show that cool WDs have redder $u-g$ colors than predicted. Our models do not include the Ly $\alpha$ far red-wing opacity (Kowalski \& Saumon 2006). Hence, the observed mismatch in the $u-g$ colors is not surprising. On the other hand, the observed range of $g-r, r-i$, and $i-z$ colors agree well with the pure hydrogen or helium atmosphere model predictions except for the WDs with significant $i$ - or $z$-band absorption. The $g-r$ versus $r-i$ diagram provides an efficient way to identify WDs that show strong absorption in the $i$-band, and the $r-i$ versus $i-z$ diagram provides a reliable selection of $\mathrm{WD}+\mathrm{dM}$ pairs.

The $u-g$ versus $g-r$ diagram reveals six DQs in the upper right corner of the figure. Strong molecular absorption in the $g$ band causes the $g-r$ colors to be redder than normal. One of these DQs, J1247+0646, is about 0.7 mag redder than the other five in our sample, and it is also redder than all other published DQ WDs ${ }^{12}$ including the extreme DQ GSC2U $\mathrm{J} 131147.2+292348$ (Carollo et al. 2003). J1247+0646 is clearly a very cool, peculiar DQ WD.

Figure 9 displays optical and near-infrared color-color diagrams for the Bergeron et al. (2001) sample and our Gemini, IRTF, and UKIRT sample of WDs. The Bergeron et al. (2001) sample includes stars with $T_{\text {eff }} \leqslant 12,000 \mathrm{~K}$. LHS 1126 (Luyten Half Second catalog; Luyten 1979) is the only star in that sample that shows significant absorption in the near-infrared. In addition to the previously known ultracool WD candidates, these

12 There are two redder DQ WDs, both with $g-i=2.3 \mathrm{mag}$, discovered in the DR7 and SEGUE observations.

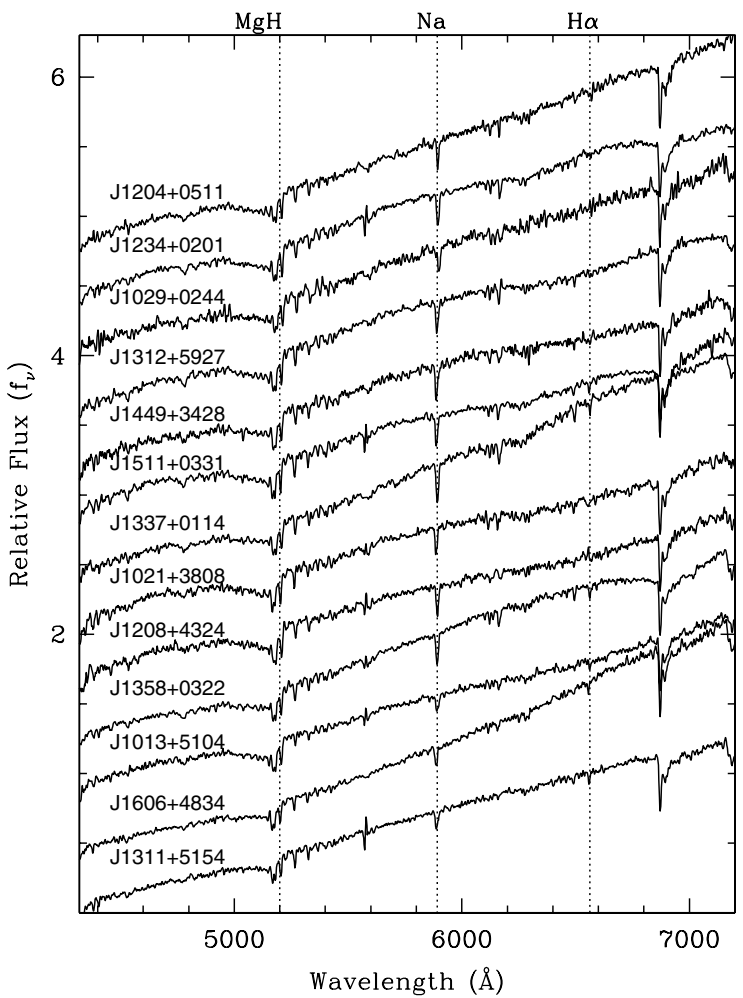

Figure 7. HET spectra of the contaminants in our survey: subdwarfs. The spectra are ordered in increasing $g-i$ color. 

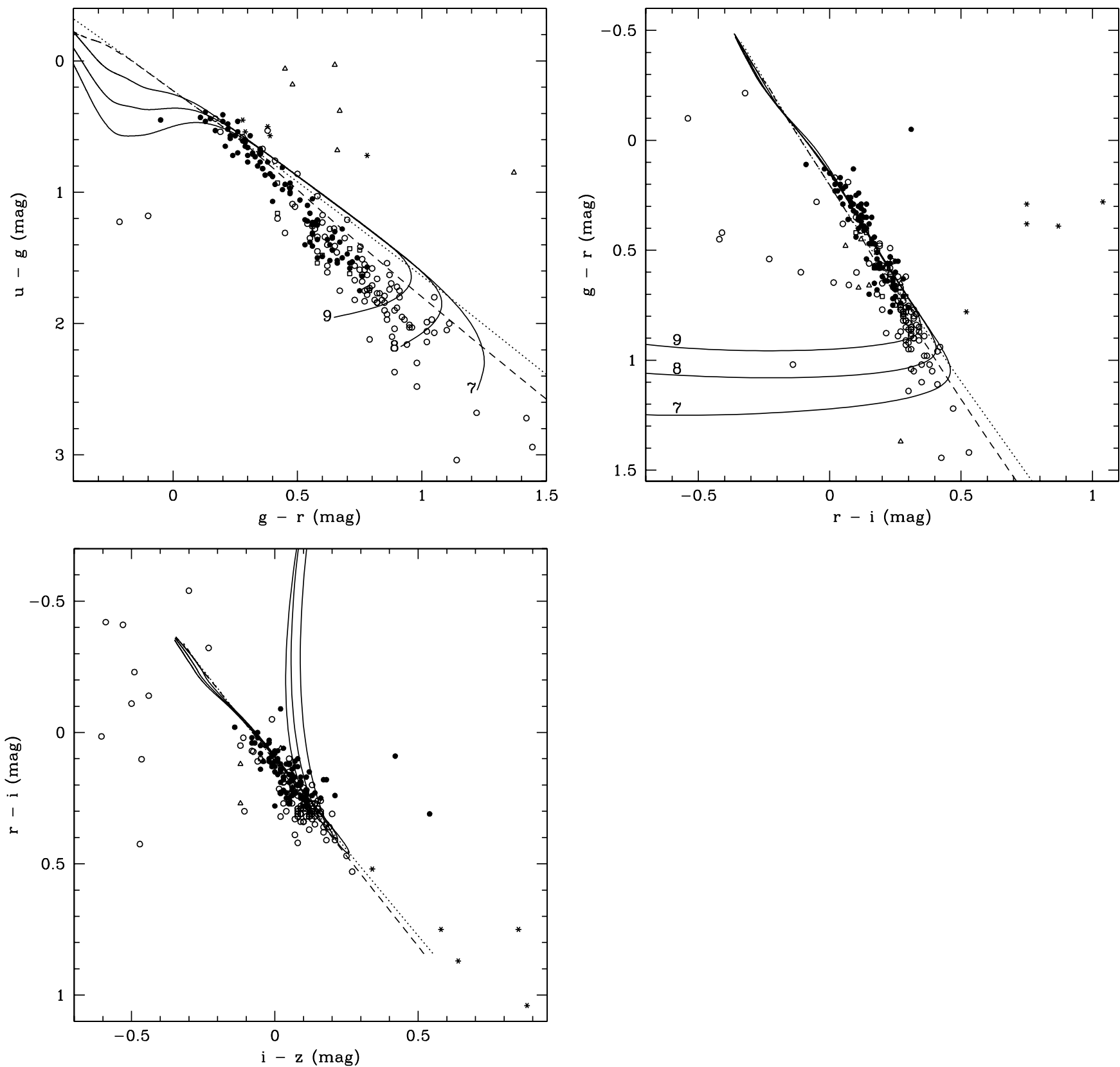

Figure 8. Color-color diagrams for DA (filled circles), DC (open circles), DQ (open triangles), DZ (open squares), WD+dM (asterisks) in the SDSS. The solid lines show the predicted colors for pure hydrogen atmosphere WDs with $T_{\text {eff }}=2000-30,000 \mathrm{~K}$ and $\log g=7,8$, and 9. The dashed line shows a pure helium atmosphere WD sequence with $T_{\text {eff }}=3000-30,000 \mathrm{~K}$ and $\log g=8$, whereas the dotted line shows the colors for blackbody SEDs for the same temperature range.

diagrams reveal eight more WDs that show significant absorption in the $H+K$ bands or only in the $K$ band. There is a large scatter in the observed infrared colors of these stars. However, the previously known and the newly found stars with flux deficits reveal, for the first time, a pattern in the $r-J$ versus $J-H$ diagram. The observed sequence for IR-faint WDs is significantly different from the pure hydrogen model sequence indicating that these WDs most likely do not have pure hydrogen atmospheres. The dotted, long-dashed, and dash-dotted lines in Figure 9 show the predicted infrared colors for mixed atmosphere WDs with $\mathrm{H} / \mathrm{He}=10,1$, and 0.01 , respectively. The similarities between the colors for these models and the IR-faint stars suggest that these stars have mixed $\mathrm{H} / \mathrm{He}$ atmospheres.

Excluding the 14 stars from the Gates et al. (2004) and Harris et al. (2008) studies, 8 out of 112 (or 7\% of) WDs in our sample show significant absorption in the infrared. Understanding the nature of these stars is important for WD cosmochronology. Prior to our study, near-infrared photometry of only a few ultracool WDs has been done (e.g., LHS 1402, LHS 3250, and SDSS J1337+0001, Bergeron \& Leggett 2002; Bergeron et al. 2005). Our Gemini photometry for the ultracool WD candidates from Gates et al. (2004) and Harris et al. (2008) shows that six of these stars have similar $r-J$ and $J-H$ colors with LHS 3250 and J1337+0001. A few other stars from the Harris et al. (2008) sample also have similar $r-J$ colors. However, only $J$-band photometry is available for these stars and therefore they are not shown in Figure 9.

\subsection{Model Atmosphere Analysis}

We use new grids of pure hydrogen and mixed $\mathrm{H} / \mathrm{He}$ composition models with $\log g=8$ and $T_{\text {eff }}=2000-7000 \mathrm{~K}$, in steps of $250 \mathrm{~K}$. In addition, we use a new pure helium 

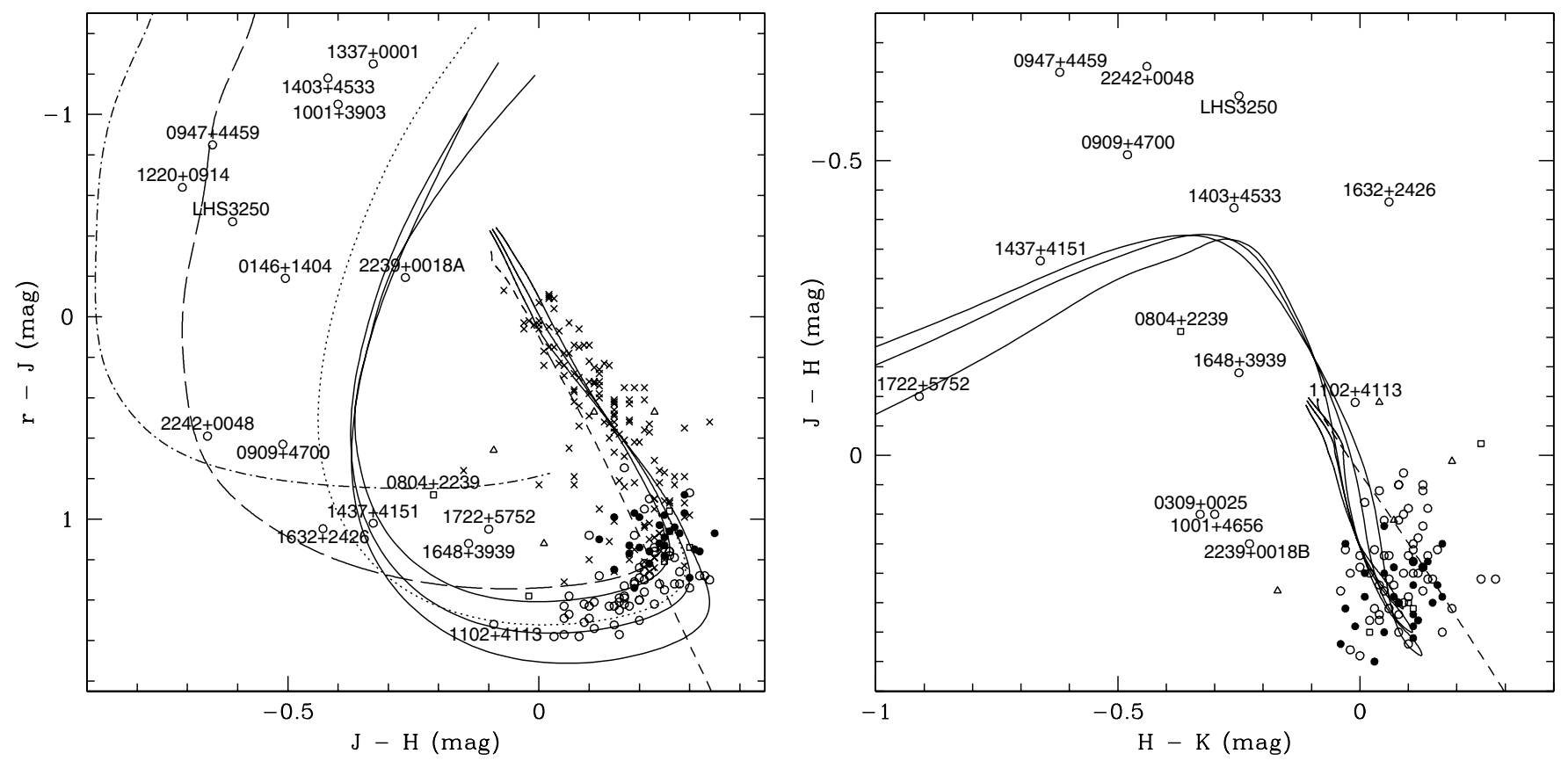

Figure 9. Near-infrared color-color diagrams for WDs studied by Bergeron et al. (2001, crosses in the left panel) and our sample (circles, triangles, and squares). The symbols are the same as in Figure 8. WDs with significant infrared flux deficits and the halo WD candidate J1102+4113 are labeled. In addition to the pure hydrogen and pure helium atmosphere model sequences, the dotted, long-dashed, and dash-dotted lines show the predicted color sequences for mixed atmosphere models with $\left(T_{\text {eff }}=2000-6000 \mathrm{~K}\right.$ and $\left.\log g=8\right) \mathrm{H} / \mathrm{He}=10,1$, and 0.01 , respectively.

atmosphere model grid with $T_{\text {eff }}=3500-7000 \mathrm{~K}$. The Hrich models are very similar to those of Bergeron et al. (2001) but with updated collision-induced absorption (CIA) opacities (see the discussion in Tremblay \& Bergeron 2007). We use a pure helium model grid with the non-ideal equation of state of Bergeron et al. (1995). The previous models calculated with this theory had a programming error. We use a corrected grid for this work. The number of free electrons in cool He-rich models is about an order of magnitude higher than the model grid used by Bergeron et al. (1997, 2001). However, the differences between the old and the new models are small, with shifts in $T_{\text {eff }}$ of $\approx 200 \mathrm{~K}$ for the coolest stars. The quality of the fits is also similar in both cases.

Our mixed atmosphere models are based on those of Bergeron \& Leggett (2002) but with three significant changes. We now include the $\mathrm{HeH}^{+}$molecule in the equation of state (Harris et al. 2004), which is the most important update here. We also include the most recent calculations for the $\mathrm{He}^{-}$opacity (John 1994). Bergeron \& Leggett (2002) included the Hummer \& Mihalas (1988) non-ideal effects only in the relative state populations, which is a very good approximation for most DA and DB WDs. However, for ultracool WDs, a full implementation of the non-ideal effects is necessary, including corrections to the pressure (or density) at each depth. For simplicity, we have neglected all non-ideal effects, since these effects are poorly understood and the Hummer \& Mihalas results are at odds with more recent calculations (Kowalski 2006). In Figure 10, we present an illustrative sequence of models at constant $T_{\text {eff }}$ and $\log g$, which shows that the maximum CIA absorption is now predicted at $\mathrm{H} / \mathrm{He} \sim 10^{-2}$ instead of $10^{-5}$ as found by Bergeron \& Leggett (2002). Thus we expect differences in the $\mathrm{H} / \mathrm{He}$ ratios of $\sim 0-3$ dex.

Our fitting technique is described at length in Bergeron et al. (2001). Briefly, we convert the magnitudes into observed fluxes using the method of Holberg \& Bergeron (2006) and

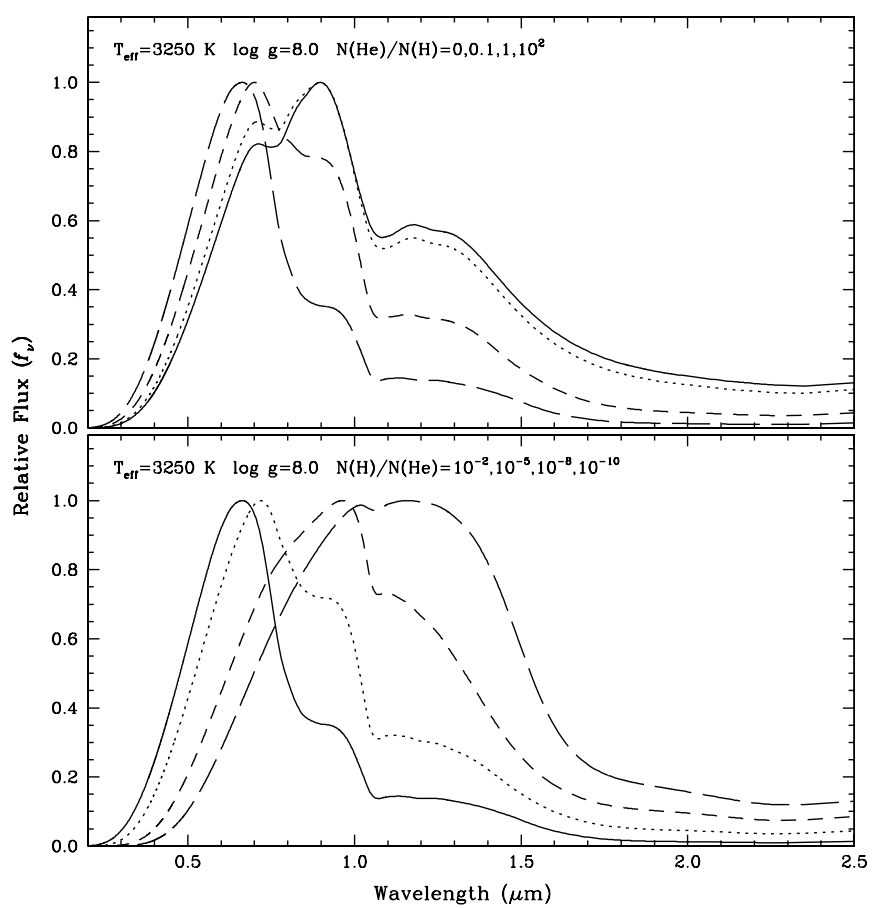

Figure 10. Similar to Figure 5 of Bergeron \& Leggett (2002) but with our updated models for mixed $\mathrm{H} / \mathrm{He}$ compositions. The top panel compares models at $T_{\text {eff }}=3250 \mathrm{~K}$ and $\log g=8.0$ from a pure hydrogen composition (solid line) to a value of $\mathrm{H} / \mathrm{He}=10^{-2}$, where the infrared flux deficiency is the strongest. In the bottom panel, the hydrogen abundance is further decreased from a value of $\mathrm{H} / \mathrm{He}=10^{-2}$ (solid line) to $10^{-10}$.

the appropriate filters. Then we fit the resulting SEDs with those predicted from model atmosphere calculations using a nonlinear least-squares method. Only $T_{\text {eff }}$ and the solid angle $\pi(R / D)^{2}$, where $R$ is the radius of the star and $D$ is its distance 
from Earth, are considered free parameters. Since no parallax measurements are available, we assume a surface gravity of $\log g=8$ (Bergeron et al. 2001).

Our models do not include the opacity due to the red wing of Ly $\alpha$ (Kowalski \& Saumon 2006). Hence, we omit the $u$-band photometry from all fits. We also omit the $g$-band photometry for stars cooler than $4600 \mathrm{~K}$ since the missing Ly $\alpha$ opacity has a larger impact at lower temperatures (see Lodieu et al. 2009). The reason for neglecting the $B$ and $V$ (or $u$ and $g$ ) filter photometry at lower temperatures is discussed at length in Bergeron et al. (1997, Section 5.2.2). The atmospheric parameters for the cool WD BPM 4729 obtained with models including this opacity ( $T_{\text {eff }}=5820 \mathrm{~K}$ and $\log g=8.30$, Kowalski \& Saumon 2006) and with models excluding this opacity and the UV filters $\left(T_{\text {eff }}=5730 \pm 110 \mathrm{~K}\right.$ and $\log g=8.21 \pm 0.09$, Bergeron et al. 2001) are essentially the same. This is because the Ly $\alpha$ opacity affects a wavelength region where there is very little flux; hence the atmospheric structure is not affected much by a change of the opacity in the UV (although the predicted colors in the UV are).

We use the SEDs together with the optical spectra at $\mathrm{H} \alpha$ to constrain the surface composition. If optical spectroscopy is unavailable, we choose the best photometric fit. We use the mixed $\mathrm{H} / \mathrm{He}$ atmosphere models only if there is a strong infrared absorption. The majority of the objects in our sample are consistent with either pure hydrogen or pure helium atmospheres, though some are best explained with mixed $\mathrm{H} / \mathrm{He}$ atmosphere models. There are almost no cases of mild absorption where mixed $\mathrm{H} / \mathrm{He}$ models would be needed. Obviously, the addition of helium into a hydrogen-rich atmosphere provides another free parameter in our model fits and it can improve the fits slightly, but these determinations are not statistically significant.

We use the Dufour et al. (2005) results for the DQ stars, but we use regular He-rich or H-rich models for the DZA and DAZ WDs. For the DQpec stars, we use the procedure of Bergeron et al. $(1997,2001)$ in which we use the normalized observed spectra to recalibrate the synthetic fluxes and take the molecular bands into account in first order. Even with this procedure the fits are poor and we removed both the $u$ - and $g$-band photometry from the fits. Table 3 presents the best-fit model temperatures, distances, ages, and compositions for our sample of $\mathrm{WDs}^{13}$ assuming $\log g=8$.

The errors in temperature are largely dominated by the observational uncertainties with a minor role from the unknown surface gravity. Other quantities (distances and ages) depend, of course, on the surface gravity assumption. The thickness of the hydrogen envelope and the core composition also play a role in age estimates. In addition, a WD may change its surface composition several times during its lifetime as a result of competing mechanisms (e.g., gravitational settling, accretion, dredge-up, and convective mixing). Hence, it is difficult to estimate the true age of a star at a given time. However, Tremblay \& Bergeron (2008) demonstrate that the majority of DA stars have relatively massive hydrogen envelopes $\left(M_{\mathrm{H}} / M_{\star} \sim 10^{-6}\right.$ or more) that prevent them from conversion to helium-dominated atmospheres. WDs with thin hydrogen layers likely end up with pure $\mathrm{He}$ or mixed $\mathrm{H} / \mathrm{He}$ atmospheres due to convective mixing. All of these issues have been thoroughly discussed in Bergeron et al. (1997, Section 6.4), Bergeron et al. (2001, Sections 2.3 and 5.5), and Fontaine et al. (2001).

\footnotetext{
13 One of the targets, J0804+2239, is a DZ WD with strong IR absorption. This star is not included in Table 3. Its intriguing SED will be discussed in a future publication.
}

\subsubsection{Pure Hydrogen and Pure Helium Atmosphere WDs}

Figure 11 shows a representative sample of fits for DA stars with $T_{\text {eff }}=5000-6000 \mathrm{~K}$. J0003-0111, J0250-0910, and $\mathrm{J} 1116+0925$ all show $\mathrm{H} \alpha$ absorption and their optical and nearinfrared SEDs are best matched by pure hydrogen atmosphere models. The spectroscopic observations of $\mathrm{H} \alpha$ are not used directly in the fitting procedure, but they serve as an internal check of our photometric solutions. The theoretical line profiles are calculated using the parameters obtained from the SED fits. This figure shows that the predicted line profiles are in good agreement with the pure hydrogen atmosphere model solutions derived from the photometric observations. On the other hand, for three of the stars in this figure $(\mathrm{J} 0330+0037$, $\mathrm{J} 1212+4345$, and $\mathrm{J} 2350-0848$ ) pure helium atmosphere models fit the optical and infrared photometry better than the pure hydrogen atmosphere models. However, the difference between pure hydrogen and pure helium model SEDs is relatively small at these temperatures and the observed photometry is also consistent with the pure hydrogen model predictions within the errors. The residuals observed in the pure hydrogen model fits are most likely due to small photometric errors or calibration problems. These small differences show the limitations of the entire photometric approach, and consequently the difficulty in assigning $\mathrm{H}$ versus $\mathrm{He}$ composition when $\mathrm{H} \alpha$ is invisible. In any case, the excellent match between the $\mathrm{H} \alpha$ line profiles and observations rules out significant amounts of helium in the atmosphere. Hence, we assume a pure hydrogen composition for all stars that show $\mathrm{H} \alpha$ absorption.

Figure 12 shows sample fits for 11 helium-rich DC stars. The spectroscopic fits are not shown here since all objects are featureless near the $\mathrm{H} \alpha$ region. Several of the stars in this figure are warm enough to show $\mathrm{H} \alpha$ if they were hydrogen rich. With the resolution and signal-to-noise ratio of our observations, we are able to detect $\mathrm{H} \alpha$ for stars hotter than $5000 \mathrm{~K}$. The lack of $\mathrm{H} \alpha$ absorption reveals a helium-rich composition, and the pure helium models provide excellent fits to the SEDs of these objects. There are 42 stars with $T_{\text {eff }} \geqslant 4530 \mathrm{~K}$ that are best explained as pure helium atmosphere objects, but there are none below this temperature. Given the observed infrared colors of cool WDs, perhaps this is not surprising. The $r-J$ versus $J-H$ color-color diagram (Figure 9) shows that the coolest WDs show absorption in the infrared, indicating that they have hydrogen in their atmospheres.

Figure 13 displays the model fits to the SEDs of the eight coolest DC WDs in our sample excluding the ultracool $\left(T_{\text {eff }}<\right.$ $4000 \mathrm{~K})$ WDs. These SEDs are best explained with pure hydrogen atmosphere models with $T_{\text {eff }}=4150-4420 \mathrm{~K}$. Omitting the $u$ - and $g$-band photometry from the fits (due to the missing Ly $\alpha$ opacity), our models are able to explain the overall SEDs of these WDs fairly well.

\subsubsection{Mixed H/He Atmosphere WDs}

Bergeron et al. (2001) do not find a large population of cool WDs with mixed hydrogen and helium atmospheres. Such stars would show up as outliers in the optical and infrared color-color diagrams due to the $\mathrm{H}_{2}-\mathrm{He} \mathrm{CIA}$, which is predicted to produce strong flux deficits in the infrared. Our sample has half a dozen new WDs with significant absorption in the infrared (Figure 9). Pure hydrogen and pure helium models fail to reproduce the SEDs for these stars. Figure 14 presents mixed $\mathrm{H} / \mathrm{He}$ atmosphere model fits to eight DC WDs in our sample. The mixed $\mathrm{H} / \mathrm{He}$ atmosphere models with $\log (\mathrm{H} / \mathrm{He})=-5.9$ to 
Table 3

Physical Parameters

\begin{tabular}{|c|c|c|c|c|c|c|c|c|}
\hline Object & $\begin{array}{l}T_{\text {eff }} \\
(\mathrm{K})\end{array}$ & $\begin{array}{c}d \\
(\mathrm{pc})\end{array}$ & $\begin{array}{c}\text { Cooling Age } \\
(\mathrm{Gyr})\end{array}$ & $\begin{array}{c}\text { Comp } \\
(\log \mathrm{H} / \mathrm{He})\end{array}$ & Type & $\begin{array}{c}\mu_{\mathrm{R} . \mathrm{A} .} \\
\left(\mathrm{mas} \mathrm{yr}^{-1}\right)\end{array}$ & $\begin{array}{c}\mu_{\text {Decl. }} \\
\left(\text { mas yr }^{-1}\right)\end{array}$ & Notes \\
\hline J0003-0111 & $5450 \pm 70$ & 72 & 3.4 & $\mathrm{H}$ & DA & 98 & -16 & $\mathrm{a}$ \\
\hline $\mathrm{J} 0033+1451$ & $5360 \pm 180$ & 53 & 4.5 & -3.4 & $\ldots$ & -209 & -190 & \\
\hline $\mathrm{J} 0045+1420$ & $5070 \pm 90$ & 54 & 5.4 & $\mathrm{H}$ & DZA & 260 & -53 & $\mathrm{~b}, \mathrm{~d}$ \\
\hline $\mathrm{J} 0102+1401$ & $4830 \pm 50$ & 54 & 6.4 & $\mathrm{He}$ & $\mathrm{DC}$ & 12 & 106 & \\
\hline J0146+1404 & $3930 \pm 235$ & 60 & 8.5 & -2.5 & $\mathrm{DC}$ & 252 & 38 & \\
\hline $\mathrm{J} 0157+1335$ & $4820 \pm 70$ & 56 & 6.4 & -5.0 & $\mathrm{DC}$ & 87 & -62 & $\mathrm{e}$ \\
\hline J0212-0040 & $6010 \pm 80$ & 79 & 2.2 & $\mathrm{H}$ & $\ldots$ & 132 & 18 & \\
\hline $\mathrm{J} 0250-0910$ & $5640 \pm 80$ & 67 & 2.8 & $\mathrm{H}$ & DA & 106 & 2 & $\mathrm{a}$ \\
\hline J0256-0700 & $4420 \pm 110$ & 35 & 7.8 & $\mathrm{H}$ & $\mathrm{DC}$ & 373 & -202 & \\
\hline J0301-0044 & $4530 \pm 50$ & 65 & 7.1 & $\mathrm{He}$ & $\mathrm{DC}$ & 108 & -532 & \\
\hline J0307-0715 & $5840 \pm 80$ & 40 & 2.4 & $\mathrm{H}$ & DA & -199 & -452 & \\
\hline $\mathrm{J} 0309+0025$ & $4920 \pm 50$ & 37 & 6.2 & -4.4 & $\mathrm{DC}$ & -6 & -106 & \\
\hline J0310-0110 & $4970 \pm 50$ & 117 & 6.1 & $\mathrm{He}$ & $\mathrm{DC}$ & -36 & -80 & \\
\hline J0314-0105 & $5800 \pm 80$ & 61 & 2.5 & $\mathrm{H}$ & DA & -77 & -71 & \\
\hline J0320-0716 & $6570 \pm 80$ & 129 & 1.8 & $\mathrm{He}$ & DQ & 120 & -11 & \\
\hline J0330+0037 & $5870 \pm 90$ & 108 & 2.4 & $\mathrm{H}$ & $\mathrm{DA}$ & 77 & 34 & $\mathrm{a}, \mathrm{c}$ \\
\hline J0406-0644 & $5960 \pm 80$ & 50 & 2.3 & $\mathrm{H}$ & DA & 67 & 27 & \\
\hline $\mathrm{J} 0747+2438 \mathrm{~N}$ & $4890 \pm 50$ & 52 & 6.3 & $\mathrm{He}$ & $\mathrm{DC}$ & 140 & -70 & $\mathrm{~b}$ \\
\hline $\mathrm{J} 0747+2438 \mathrm{~S}$ & $5740 \pm 80$ & 54 & 2.6 & $\mathrm{H}$ & DA & 137 & -69 & $\mathrm{a}$ \\
\hline $\mathrm{J} 0753+4230$ & $4650 \pm 100$ & 24 & 7.1 & $\mathrm{H}$ & $\mathrm{DC}$ & 113 & -403 & \\
\hline $\mathrm{J} 0817+2451$ & $5520 \pm 70$ & 81 & 3.9 & $\mathrm{He}$ & $\ldots$ & 81 & -211 & \\
\hline $\mathrm{J} 0817+2822$ & $4720 \pm 40$ & 50 & 6.7 & $\mathrm{He}$ & $\mathrm{DC}$ & 63 & -205 & \\
\hline $\mathrm{J} 0819+3159$ & $4700 \pm 40$ & 56 & 6.7 & $\mathrm{He}$ & $\mathrm{DC}$ & 211 & -22 & \\
\hline $\mathrm{J} 0821+3727$ & $5060 \pm 60$ & 52 & 5.4 & $\mathrm{H}$ & DA & 165 & -154 & $\mathrm{a}, \mathrm{c}$ \\
\hline J0825+2841 & $5600 \pm 70$ & 66 & 2.9 & $\mathrm{H}$ & $\ldots$ & -114 & -110 & \\
\hline $\mathrm{J} 0825+5049$ & $4690 \pm 40$ & 46 & 6.7 & $\mathrm{He}$ & $\mathrm{DC}$ & -331 & -330 & \\
\hline J0836+4556 & $4880 \pm 60$ & 70 & 6.3 & $\mathrm{He}$ & $\mathrm{DC}$ & -64 & -169 & \\
\hline J0838+2804 & $5170 \pm 60$ & 58 & 5.4 & $\mathrm{He}$ & $\mathrm{DC}$ & 63 & -200 & \\
\hline J0853+4118 & $5590 \pm 70$ & 78 & 3.6 & $\mathrm{He}$ & $\ldots$ & -128 & 103 & \\
\hline J0854+3503 & $4070 \pm 130$ & 57 & 8.8 & 1.0 & $\mathrm{DC}$ & -133 & -179 & \\
\hline $\mathrm{J} 0855+3700$ & $5660 \pm 70$ & 42 & 2.8 & $\mathrm{H}$ & $\ldots$ & 148 & -89 & \\
\hline J0904+3403 & $5350 \pm 40$ & 53 & 4.6 & $\mathrm{He}$ & $\mathrm{DC}$ & -180 & -291 & \\
\hline J0909+4700 & $4510 \pm 160$ & 53 & 7.1 & -3.4 & $\mathrm{DC}$ & -117 & -179 & \\
\hline $\mathrm{J} 0933+3743$ & $5430 \pm 60$ & 68 & 4.2 & $\mathrm{He}$ & DZA & 134 & -113 & \\
\hline J0942+4437 & $4450 \pm 90$ & 42 & 7.8 & $\mathrm{H}$ & $\mathrm{DC}$ & -135 & -189 & \\
\hline J0943+5134 & $4880 \pm 60$ & 43 & 6.3 & $\mathrm{He}$ & $\mathrm{DC}$ & 131 & -261 & \\
\hline $\mathrm{J} 0947+4459$ & $3410 \pm 90$ & 39 & 9.5 & -2.7 & $\mathrm{DC}$ & 74 & 45 & \\
\hline J0947+4500 & $5100 \pm 130$ & 61 & 5.2 & $\mathrm{H}$ & DA & 74 & 45 & $\mathrm{~b}$ \\
\hline $\mathrm{J} 1000+4236$ & $4840 \pm 50$ & 57 & 6.4 & $\mathrm{He}$ & $\mathrm{DC}$ & -342 & -80 & \\
\hline $\mathrm{J} 1001+3903$ & $3050 \pm 150$ & 45 & 10.2 & -2.9 & $\mathrm{DC}$ & -301 & -185 & \\
\hline $\mathrm{J} 1001+4656$ & $4150 \pm 70$ & 33 & 8.6 & $\mathrm{H}$ & $\mathrm{DC}$ & -17 & -339 & \\
\hline $\mathrm{J} 1002+6108$ & $4420 \pm 120$ & 39 & 7.8 & $\mathrm{H}$ & $\mathrm{DC}$ & -448 & -328 & \\
\hline $\mathrm{J} 1042+4932$ & $5380 \pm 60$ & 66 & 4.5 & $\mathrm{He}$ & $\mathrm{DC}$ & -120 & -85 & \\
\hline $\mathrm{J} 1102+6707$ & $5080 \pm 60$ & 68 & 5.7 & $\mathrm{He}$ & $\mathrm{DC}$ & -380 & -185 & \\
\hline $\mathrm{J} 1102+4030$ & $5110 \pm 50$ & 49 & 5.2 & $\mathrm{H}$ & DA & 193 & -256 & $\mathrm{~b}, \mathrm{c}$ \\
\hline $\mathrm{J} 1104+0436$ & $4400 \pm 120$ & 49 & 7.9 & $\mathrm{H}$ & $\mathrm{DC}$ & 101 & -385 & \\
\hline $\mathrm{J} 1107+4855$ & $4640 \pm 160$ & 46 & 7.1 & $\mathrm{H}$ & $\mathrm{DC}$ & -726 & -79 & \\
\hline $\mathrm{J} 1116+0925$ & $5470 \pm 90$ & 63 & 3.3 & $\mathrm{H}$ & DA & 7 & -152 & $\mathrm{a}$ \\
\hline $\mathrm{J} 1117+5010$ & $4900 \pm 60$ & 53 & 6.3 & $\mathrm{He}$ & $\mathrm{DC}$ & 170 & -120 & \\
\hline $\mathrm{J} 1119-0107$ & $4760 \pm 50$ & 64 & 6.6 & $\mathrm{He}$ & $\mathrm{DC}$ & -291 & -28 & \\
\hline $\mathrm{J} 1130+1002$ & $5230 \pm 70$ & 52 & 4.5 & $\mathrm{H}$ & DA & 145 & -311 & $\mathrm{a}, \mathrm{c}$ \\
\hline $\mathrm{J} 1147+4303$ & $4900 \pm 30$ & 48 & 6.2 & $\mathrm{He}$ & $\mathrm{DC}$ & 114 & -174 & \\
\hline $\mathrm{J} 1151+1253$ & $5260 \pm 70$ & 69 & 5.0 & $\mathrm{He}$ & $\mathrm{DC}$ & 4 & -210 & \\
\hline $\mathrm{J} 1158+0004$ & $4390 \pm 100$ & 30 & 7.9 & $\mathrm{H}$ & $\mathrm{DC}$ & -17 & 182 & \\
\hline $\mathrm{J} 1202-0313$ & $4490 \pm 160$ & 55 & 7.6 & $\mathrm{H}$ & $\mathrm{DC}$ & -73 & 134 & \\
\hline $\mathrm{J} 1204+6222$ & $4820 \pm 50$ & 48 & 6.4 & $\mathrm{He}$ & $\mathrm{DC}$ & -21 & -159 & \\
\hline $\mathrm{J} 1212+4345$ & $5450 \pm 70$ & 73 & 3.4 & $\mathrm{H}$ & DA & -210 & 59 & $\mathrm{a}, \mathrm{c}$ \\
\hline $\mathrm{J} 1212+0440$ & $4450 \pm 110$ & 55 & 7.8 & $\mathrm{H}$ & $\mathrm{DC}$ & -279 & -72 & \\
\hline $\mathrm{J} 1220+0914$ & $2950 \pm 130$ & 39 & 10.5 & -5.1 & $\mathrm{DC}$ & -341 & -372 & \\
\hline $\mathrm{J} 1220+4519$ & $5570 \pm 80$ & 81 & 3.0 & $\mathrm{H}$ & $\ldots$ & -203 & -21 & \\
\hline $\mathrm{J} 1234+0109$ & $5500 \pm 80$ & 92 & 3.2 & $\mathrm{H}$ & $\mathrm{DA}$ & -284 & -55 & $\mathrm{a}$ \\
\hline $\mathrm{J} 1234+6605$ & $5430 \pm 90$ & 70 & 3.5 & $\mathrm{H}$ & DA & -185 & -72 & $\mathrm{a}, \mathrm{c}$ \\
\hline $\mathrm{J} 1238+3502$ & $2290 \pm 120$ & 36 & 12.1 & -7.8 & $\mathrm{DC}$ & -130 & -124 & \\
\hline $\mathrm{J} 1247+0646$ & $5120 \pm 180$ & 60 & 5.6 & -0.7 & DQpec & -382 & 71 & \\
\hline $\mathrm{J} 1251+4403$ & $3200 \pm 60$ & 66 & 10.0 & -2.3 & $\mathrm{DC}$ & -167 & 30 & \\
\hline
\end{tabular}


Table 3

(Continued)

\begin{tabular}{|c|c|c|c|c|c|c|c|c|}
\hline Object & $\begin{array}{l}T_{\text {eff }} \\
(\mathrm{K}) \\
\end{array}$ & $\begin{array}{c}d \\
(\mathrm{pc}) \\
\end{array}$ & $\begin{array}{l}\text { Cooling Age } \\
(\mathrm{Gyr})\end{array}$ & $\begin{array}{c}\text { Comp } \\
(\log \mathrm{H} / \mathrm{He})\end{array}$ & Type & $\begin{array}{c}\mu_{\text {R.A. }} \\
\left(\mathrm{mas} \mathrm{yr}^{-1}\right)\end{array}$ & $\begin{array}{c}\mu_{\text {Decl. }} \\
\left(\operatorname{mas~yr}^{-1}\right)\end{array}$ & Notes \\
\hline $\mathrm{J} 1255+4655$ & $4580 \pm 160$ & 41 & 7.3 & $\mathrm{H}$ & DC & -1089 & -114 & \\
\hline $\mathrm{J} 1259+0442$ & $4840 \pm 50$ & 53 & 6.4 & $\mathrm{He}$ & DC & 201 & -59 & \\
\hline $\mathrm{J} 1313+0226$ & $4360 \pm 90$ & 29 & 8.0 & $\mathrm{H}$ & DC & -744 & -116 & \\
\hline $\mathrm{J} 1317+0621$ & $5400 \pm 70$ & 49 & 3.6 & $\mathrm{H}$ & DA & 248 & -152 & $\mathrm{a}, \mathrm{c}, \mathrm{f}$ \\
\hline $\mathrm{J} 1322-0050$ & $4840 \pm 80$ & 42 & 6.4 & $\mathrm{He}$ & DC & -156 & 118 & \\
\hline $\mathrm{J} 1324+4619$ & $5410 \pm 70$ & 62 & 3.6 & $\mathrm{H}$ & $\ldots$ & 67 & -158 & \\
\hline $\mathrm{J} 1340+4033$ & $4770 \pm 60$ & 52 & 6.6 & $\mathrm{He}$ & DC & 76 & -195 & \\
\hline $\mathrm{J} 1347+5036$ & $5210 \pm 70$ & 58 & 4.6 & $\mathrm{H}$ & DA & 166 & -178 & $\mathrm{a}$ \\
\hline $\mathrm{J} 1352+0907$ & $5150 \pm 50$ & 61 & 5.5 & $\mathrm{He}$ & DC & 113 & -305 & \\
\hline $\mathrm{J} 1358+3704$ & $5810 \pm 100$ & 89 & 2.5 & $\mathrm{H}$ & DA & 86 & -59 & $\mathrm{a}$ \\
\hline $\mathrm{J} 1403+4533$ & $2670 \pm 1500$ & 24 & 11.1 & -3.0 & DC & -271 & -84 & \\
\hline $\mathrm{J} 1405+1414$ & $5360 \pm 60$ & 71 & 4.5 & $\mathrm{He}$ & $\ldots$ & -26 & -105 & \\
\hline $\mathrm{J} 1416+0752$ & $5480 \pm 60$ & 76 & 4.0 & $\mathrm{He}$ & $\ldots$ & 0 & -92 & \\
\hline $\mathrm{J} 1422+0459$ & $4430 \pm 110$ & 43 & 7.8 & $\mathrm{H}$ & DC & -277 & -62 & \\
\hline $\mathrm{J} 1426+0937$ & $5200 \pm 70$ & 67 & 5.2 & $\mathrm{He}$ & DC & -180 & -8 & \\
\hline $\mathrm{J} 1436+4332$ & $4750 \pm 40$ & 26 & 6.6 & $\mathrm{He}$ & DC & -314 & 505 & \\
\hline $\mathrm{J} 1437+4151$ & $4480 \pm 50$ & 44 & 7.2 & -4.3 & DC & -157 & -69 & \\
\hline $\mathrm{J} 1442+4013$ & $5740 \pm 430$ & 81 & 3.1 & -2.7 & DQpec & -199 & -89 & \\
\hline $\mathrm{J} 1442+5546$ & $5500 \pm 90$ & 77 & 3.2 & $\mathrm{H}$ & DA & 91 & 0 & $\mathrm{a}$ \\
\hline $\mathrm{J} 1447+5427$ & $4890 \pm 80$ & 55 & 6.3 & $\mathrm{He}$ & DC & -231 & 39 & \\
\hline $\mathrm{J} 1452-0051$ & $5640 \pm 80$ & 78 & 2.8 & $\mathrm{H}$ & $\ldots$ & 92 & -168 & \\
\hline $\mathrm{J} 1452+4522$ & $5780 \pm 50$ & 115 & 3.0 & $\mathrm{He}$ & DC & -54 & 76 & \\
\hline $\mathrm{J} 1456+0853$ & $5420 \pm 60$ & 68 & 4.3 & $\mathrm{He}$ & $\ldots$ & 19 & -138 & \\
\hline $\mathrm{J} 1458+1146$ & $4780 \pm 50$ & 39 & 6.5 & $\mathrm{He}$ & DC & -131 & -94 & \\
\hline J1526-0007 & $5440 \pm 50$ & 74 & 3.4 & $\mathrm{H}$ & $\ldots$ & 54 & -132 & \\
\hline $\mathrm{J} 1528+3254$ & $5210 \pm 50$ & 64 & 5.2 & $\mathrm{He}$ & DC & 136 & -103 & \\
\hline $\mathrm{J} 1534+0711$ & $5320 \pm 70$ & 61 & 4.0 & $\mathrm{H}$ & DA & 126 & 114 & $\mathrm{a}, \mathrm{c}$ \\
\hline $\mathrm{J} 1534+4649$ & $4340 \pm 80$ & 27 & 8.1 & $\mathrm{H}$ & DC & -465 & 220 & \\
\hline $\mathrm{J} 1600+0019$ & $5410 \pm 40$ & 61 & 4.3 & $\mathrm{He}$ & $\ldots$ & -122 & -29 & \\
\hline $\mathrm{J} 1606+2547$ & $4880 \pm 50$ & 50 & 6.3 & $\mathrm{He}$ & DZA & -225 & -125 & \\
\hline $\mathrm{J} 1608+4235$ & $4990 \pm 70$ & 60 & 6.0 & $\mathrm{He}$ & $\mathrm{DC}$ & -123 & 102 & \\
\hline $\mathrm{J} 1615+4449$ & $4780 \pm 80$ & 56 & 6.6 & $\mathrm{H}$ & $\mathrm{DC}$ & 44 & -237 & \\
\hline $\mathrm{J} 1627+4859$ & $5330 \pm 70$ & 64 & 4.0 & $\mathrm{H}$ & DZA & -91 & 77 & $\mathrm{a}$ \\
\hline $\mathrm{J} 1632+2426$ & $4160 \pm 40$ & 44 & 8.0 & -5.6 & $\mathrm{DC}$ & -10 & -340 & \\
\hline J1648+3939 & $4850 \pm 70$ & 48 & 6.4 & -4.4 & DC & -126 & 0 & \\
\hline $\mathrm{J} 1704+3608$ & $4850 \pm 30$ & 39 & 6.4 & $\mathrm{He}$ & DC & 186 & -175 & \\
\hline $\mathrm{J} 1722+2848$ & $5370 \pm 70$ & 66 & 3.8 & $\mathrm{H}$ & DA & 2 & -255 & $\mathrm{a}, \mathrm{f}$ \\
\hline $\mathrm{J} 1722+5752$ & $4620 \pm 80$ & 54 & 6.9 & -4.2 & DC & -37 & 390 & \\
\hline J2041-0520 & $4910 \pm 30$ & 52 & 6.2 & $\mathrm{He}$ & DC & -149 & -29 & \\
\hline $\mathrm{J} 2042+0031$ & $4680 \pm 40$ & 60 & 6.8 & $\mathrm{He}$ & DC & -71 & -244 & \\
\hline J2045+0037 & $6110 \pm 100$ & 119 & 2.1 & $\mathrm{H}$ & DA & 32 & -32 & $\mathrm{a}$ \\
\hline $\mathrm{J} 2045-0710$ & $4930 \pm 70$ & 53 & 6.0 & $\mathrm{H}$ & DC & -73 & -134 & $\mathrm{~b}, \mathrm{c}$ \\
\hline J2053-0702 & $6390 \pm 40$ & 96 & 2.1 & $\mathrm{He}$ & DQ & 21 & -105 & \\
\hline J2116-0724 & $4600 \pm 100$ & 29 & 7.3 & $\mathrm{H}$ & DC & 111 & -223 & \\
\hline J2118-0737 & $4220 \pm 70$ & 56 & 8.4 & $\mathrm{H}$ & DC & 115 & -144 & \\
\hline $\mathrm{J} 2125-0734$ & $6120 \pm 100$ & 126 & 2.1 & $\mathrm{H}$ & DA & 64 & 13 & $\mathrm{a}$ \\
\hline $\mathrm{J} 2147+1127$ & $4770 \pm 80$ & 46 & 6.7 & $\mathrm{H}$ & DA & 103 & -254 & $\mathrm{~b}, \mathrm{c}$ \\
\hline $\mathrm{J} 2151-0731$ & $5620 \pm 80$ & 63 & 2.8 & $\mathrm{H}$ & $\ldots$ & 117 & 30 & \\
\hline J2204-0109 & $4750 \pm 50$ & 70 & 6.6 & $\mathrm{He}$ & DC & 112 & -303 & \\
\hline $\mathrm{J} 2239+0018 \mathrm{~A}$ & $3740 \pm 120$ & 60 & 8.9 & -3.1 & DC & 7 & 98 & \\
\hline $\mathrm{J} 2239+0018 \mathrm{~B}$ & $4440 \pm 80$ & 79 & 7.8 & $\mathrm{H}$ & DC & 7 & 98 & \\
\hline $\mathrm{J} 2242+0048$ & $3480 \pm 60$ & 32 & 9.4 & -5.9 & DC & 132 & -76 & \\
\hline $\mathrm{J} 2254+1323$ & $4390 \pm 80$ & 40 & 7.9 & $\mathrm{H}$ & DC & 329 & -199 & \\
\hline $\mathrm{J} 2307+1400$ & $5940 \pm 90$ & 84 & 2.3 & $\mathrm{H}$ & $\ldots$ & -122 & -38 & \\
\hline $\mathrm{J} 2321+0102 \mathrm{~S}$ & $5750 \pm 100$ & 82 & 2.6 & $\mathrm{H}$ & DA & -104 & -255 & $\mathrm{c}$ \\
\hline $\mathrm{J} 2321+0102 \mathrm{~N}$ & $4430 \pm 100$ & 51 & 7.8 & $\mathrm{H}$ & DC & -106 & -258 & \\
\hline $\mathrm{J} 2325+1403$ & $5030 \pm 60$ & 15 & 5.5 & $\mathrm{H}$ & DA & 336 & 115 & \\
\hline $\mathbf{J} 2330+0028$ & $5130 \pm 130$ & 67 & 5.0 & $\mathrm{H}$ & DC & 151 & 91 & \\
\hline $\mathrm{J} 2342-1001$ & $5160 \pm 90$ & 50 & 4.9 & $\mathrm{H}$ & DA & -28 & -95 & $\mathrm{a}, \mathrm{f}$ \\
\hline $\mathrm{J} 2350-0846$ & $5600 \pm 110$ & 70 & 2.9 & $\mathrm{H}$ & DA & 209 & -139 & $\mathrm{a}, \mathrm{c}$ \\
\hline
\end{tabular}

Notes. (a) $\mathrm{H} \alpha$ is clearly visible and in good agreement with the best-fit pure $\mathrm{H}$ model; (b) $\mathrm{H} \alpha$ is barely visible and cannot be confirmed; (c) the pure helium fit is significantly better; (d) the object has to be $\mathrm{H}$ rich for the $\mathrm{H} \alpha$ line to be visible at this temperature; (e) the fit is poor and unusual, and the photometry seems offset; (f) mild infrared absorption could be fitted with mixed models but the $\mathrm{H} \alpha$ line would be incompatible with predictions. 

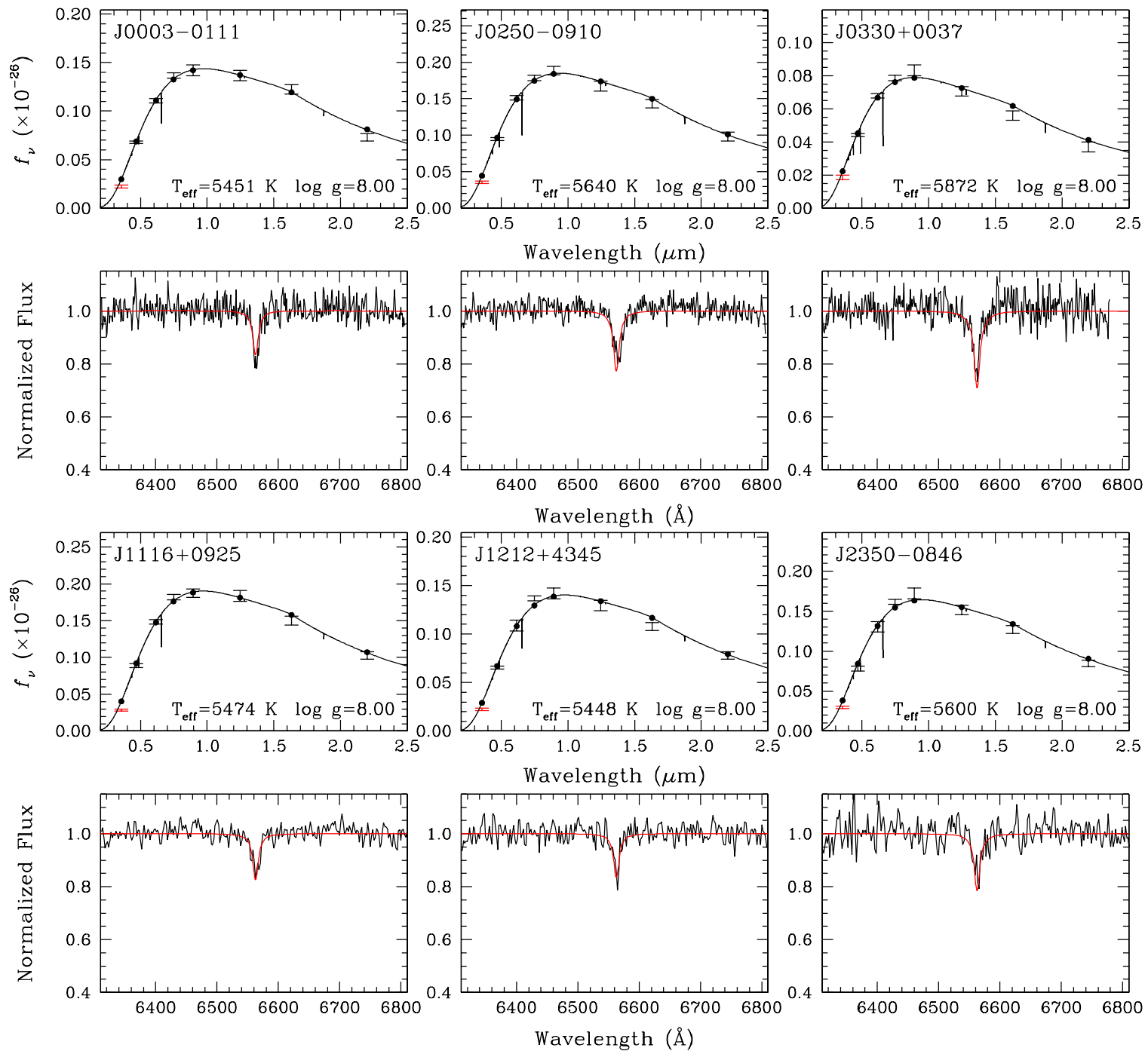

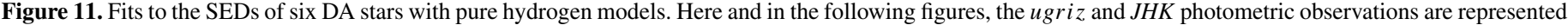

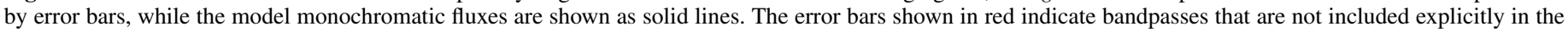

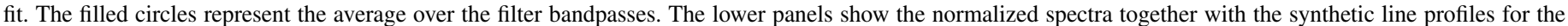
parameters obtained from the SED fits.

(A color version of this figure is available in the online journal.)

-3.4 fit the observed SEDs relatively well for these stars. Six of these targets have temperatures in the range 4500-5000 K where there are many helium-rich DC WDs. An important implication of these temperature assignments is that not all WDs that show infrared flux deficits are ultracool (have temperatures below $4000 \mathrm{~K})$. Therefore, the classification of ultracool WDs based on photometry alone (without a detailed model atmosphere analysis) can be misleading. A more appropriate term for WDs that show flux deficits in the infrared may be "IR-faint."

The best-fit model for one of the stars presented in Figure 14, $\mathbf{J} 2242+0048$, implies a temperature of $3480 \mathrm{~K}$. This star is also observed in the UKIRT Infrared Deep Sky Survey. Based on the rizYJH photometry, Lodieu et al. (2009) find a temperature of $3820 \mathrm{~K}$ with a composition of equal amounts of hydrogen and helium. Our Gemini photometry is consistent with the UKIDSS data within the errors, but we obtain a lower temperature and a helium-dominated atmosphere using grizJHK data. There are generally two solutions for IR-faint objects. This is because the CIA opacity peaks around $\log (\mathrm{H} / \mathrm{He})=-2$ and there are usually two good solutions above and below this peak with slightly different temperatures. One of the solutions is usually better than the other one. The cooler solution with a temperature of $3480 \mathrm{~K}$ explains the overall SED significantly better than the warmer solution (of Lodieu et al. 2009) over the 0.4-2.2 $\mu \mathrm{m}$ range. Clearly, J2242+0048 is one of the coolest WDs known.

\subsubsection{Peculiar $D Q W D s$}

Our optical and infrared photometry sample includes two normal and two peculiar DQs presented in Figure 3. Dufour et al. (2005) presented a detailed model atmosphere analysis of the DQ WDs in the SDSS Data Release 1 area based on photometric and spectroscopic observations. The two normal DQs in our sample are included in their study. We adopt their best-fit solutions for these two stars. J0320-0716 and J2053-0702 have best-fit temperatures of 6390 and $6570 \mathrm{~K}$ and $\log (\mathrm{C} / \mathrm{He})=-4.88$ and -5.25 , respectively (Dufour et al. 2005).

There is considerable interest in understanding the nature of the peculiar DQ WDs. The model fits to the optical and near-infrared SEDs of peculiar DQs indicate mixed $\mathrm{H} / \mathrm{He} / \mathrm{C}$ atmospheres (Bergeron et al. 1994). Figure 15 presents our model fits to the optical and near-infrared SEDs of the two 

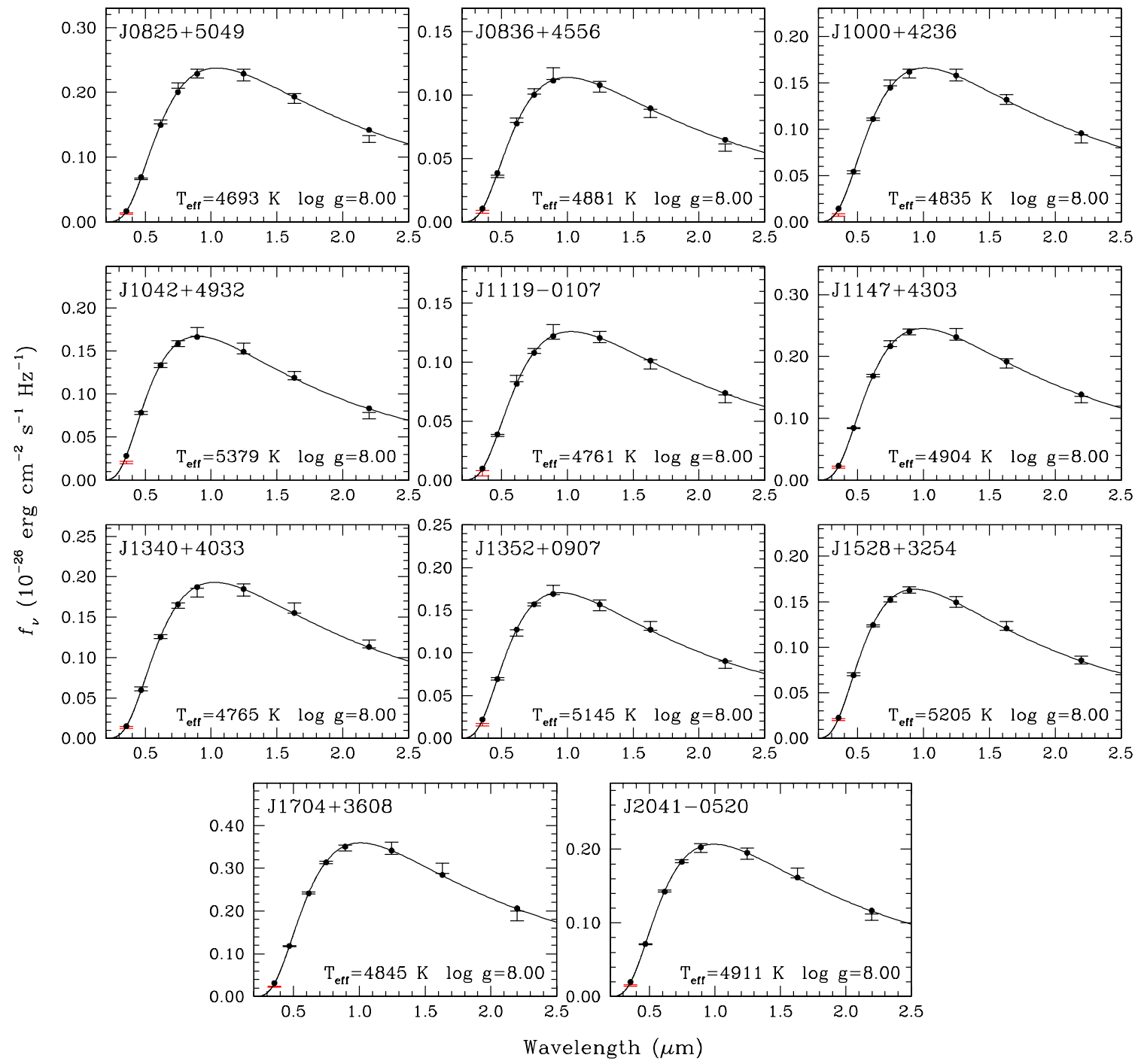

Figure 12. Fits to the SEDs of 11 DC WDs with pure helium models (filled circles). All objects have featureless spectra near the $\mathrm{H} \alpha$ region. (A color version of this figure is available in the online journal.)

DQpec WDs in our sample. J1442+4013 is a $T_{\text {eff }}=5740 \mathrm{~K}$ WD with $\log (\mathrm{H} / \mathrm{He})=-2.7$ and $\mathrm{J} 1247+0646$ is a $T_{\text {eff }}=$ $5120 \mathrm{~K}$ WD with $\log (\mathrm{H} / \mathrm{He})=-0.7$. Hall \& Maxwell (2008) argue that the molecular bands observed in peculiar DQs are most likely pressure shifted $\mathrm{C}_{2}$ bands in a helium-rich atmosphere. However, the coolest known DQpec J1247+0646 and the coolest known DQ WDs GSC2U J131147.2+292348 and SDSS J080843.15+464028.7 have essentially the same temperature (5120-5140 K; Carollo et al. 2003; Dufour et al. 2005). Normal DQ stars (including the coolest DQs) with helium-dominated, high-pressure atmospheres do not show pressure shifts. The only difference between these two classes of DQ stars (other than the shifted bands) seems to be infrared absorption, most likely from CIA due to molecular hydrogen. The peculiar DQ WDs should have atmospheric pressures that are much lower than the normal DQs as they have obviously larger opacities, contradicting the scenario proposed by Hall \& Maxwell (2008). The observed mid-infrared flux deficits for the peculiar DQ LHS 1126 demonstrate that the mixed H/He models have problems in the mid-infrared (Kilic et al. 2006b, 2008), but mixed $\mathrm{H} / \mathrm{He}$ atmospheres remain the best explanation for the observed SEDs for these WDs. Clearly, these objects deserve further investigation.

\subsubsection{Ultracool WDs}

WDs cooler than about $4000 \mathrm{~K}$ can be classified as ultracool. Starting with Hambly et al. (1999) and Harris et al. (1999), the SDSS and various other proper motion surveys have discovered ultracool WDs. LHS 1402 (Oppenheimer et al. 2001), LHS 3250 (Harris et al. 1999), and SDSS J1337+00 (Harris et al. 2001) are the best studied ultracool WDs with significant absorption in the optical and infrared. Bergeron \& Leggett (2002) and Bergeron et al. (2005) performed detailed model atmosphere analysis of these three stars using $B V R I$ and $J H(K)$ photometry. While none of their fits reproduce the SEDs perfectly, they rule out pure hydrogen composition based on the non-detection of the CIA feature near $0.8 \mu \mathrm{m}$. Instead, the SEDs are better fit with helium-dominated atmosphere models with small amounts of hydrogen. They find best-fit $T_{\text {eff }}=3240-3480 \mathrm{~K}$ and $\log (\mathrm{H} / \mathrm{He})=-3.8$ to -4.7 for these stars.

We include all 13 ultracool WD candidates discovered by Gates et al. (2004) and Harris et al. (2008; see also Vidrih et al. 2007) in our sample. We now have a chance to increase the sample of well-studied ultracool WDs significantly. The optical and infrared color-color diagrams show that several of these ultracool WDs have colors similar to the previously studied 


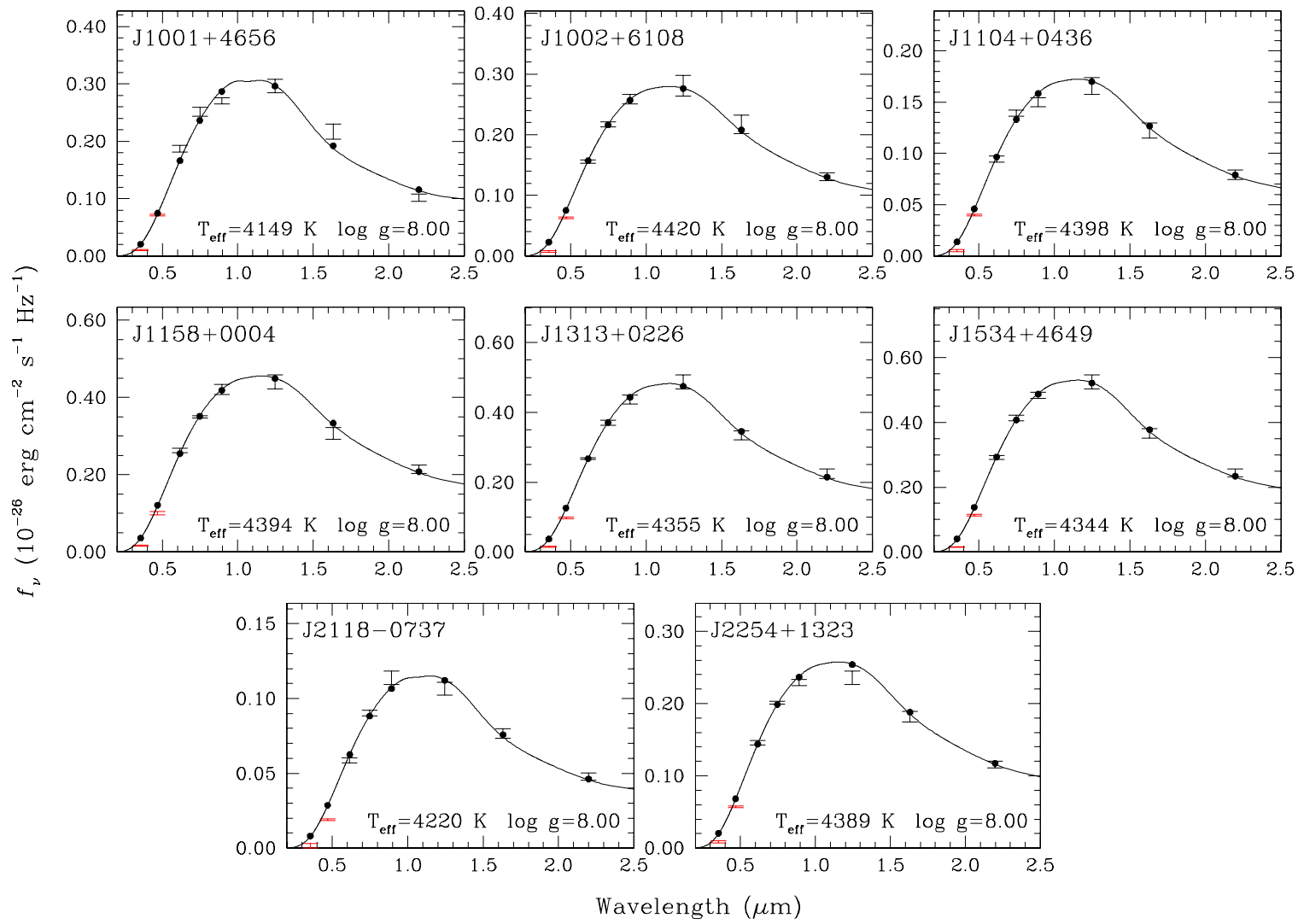

Figure 13. Fits to the SEDs of the coolest eight DC WDs in our sample, excluding the ultracool WDs. All objects have featureless spectra near the H $\alpha$ region, and the SEDs are best explained with pure hydrogen atmosphere models.

(A color version of this figure is available in the online journal.)

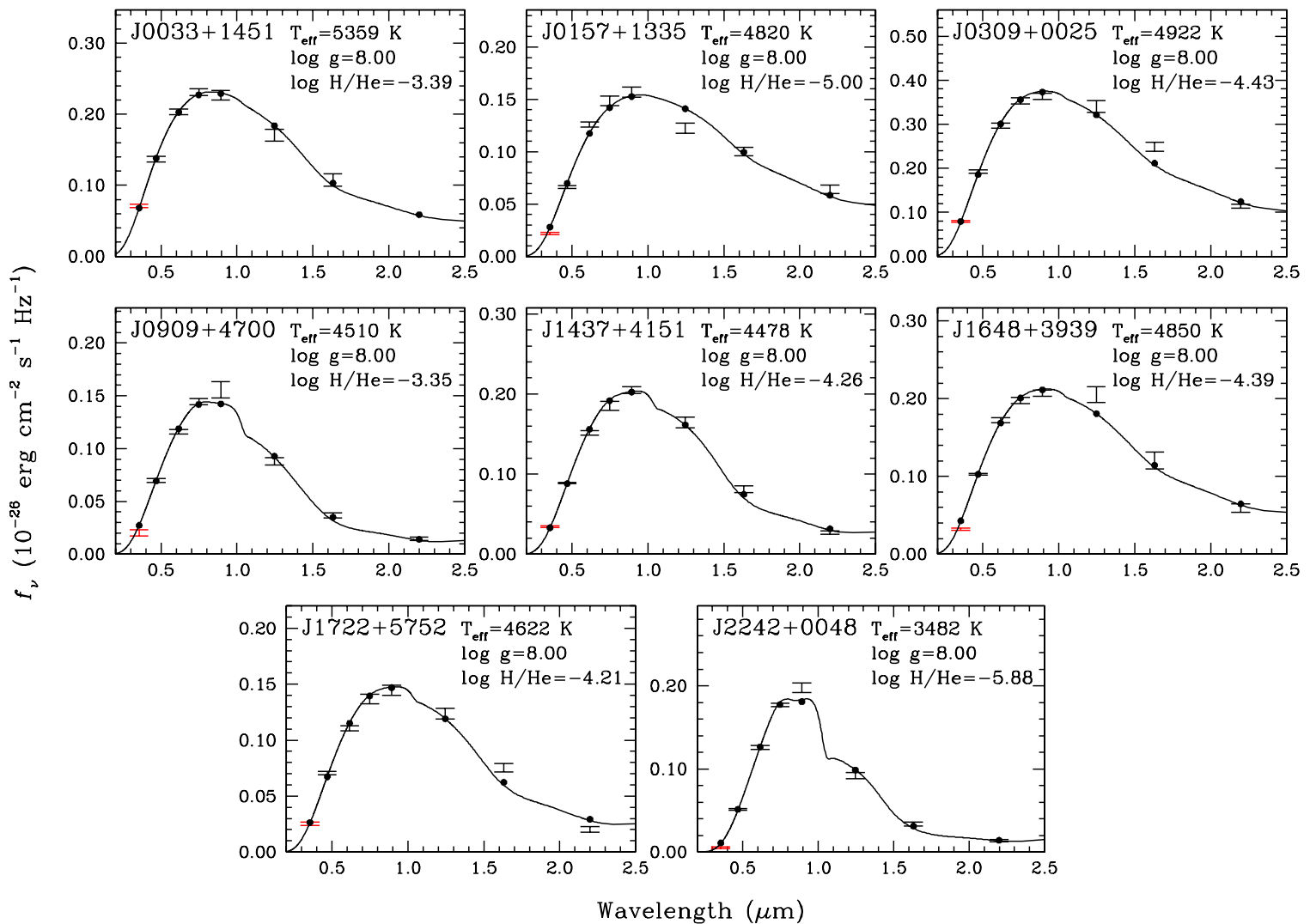

Figure 14. Fits to the SEDs of eight DC WDs with mixed $\mathrm{H} / \mathrm{He}$ atmosphere models.

(A color version of this figure is available in the online journal.) 

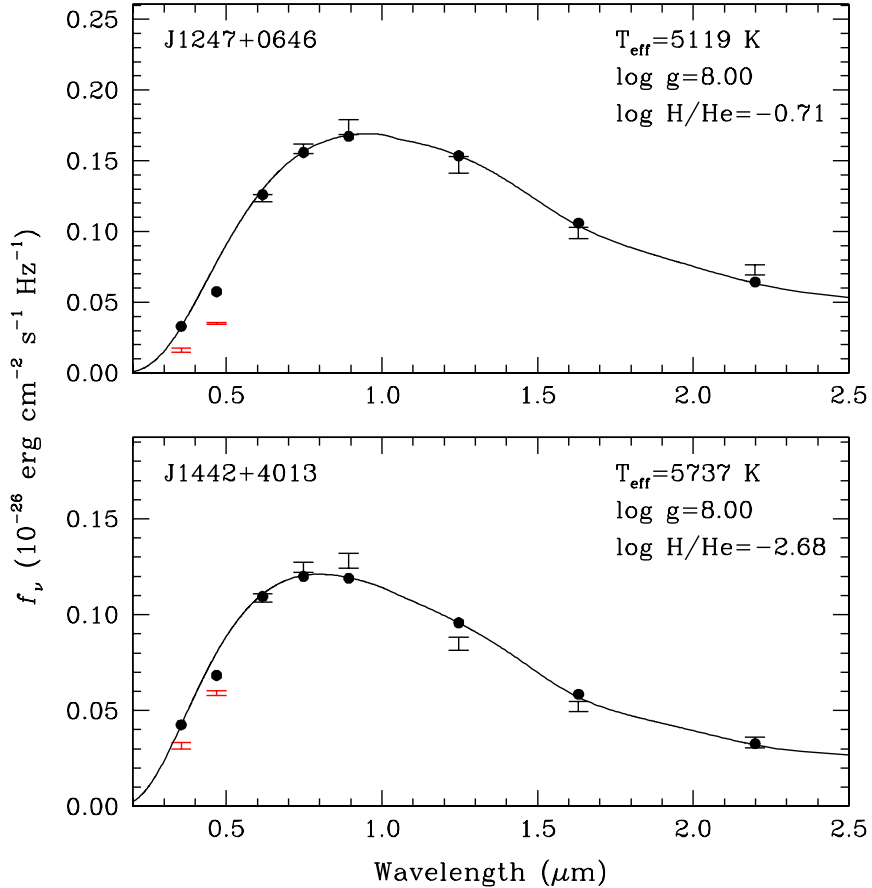

Figure 15. Fits to the SEDs of two peculiar DQ WDs with mixed $\mathrm{H} / \mathrm{He}$ atmosphere models.

(A color version of this figure is available in the online journal.)

WDs LHS 3250 and SDSS J1337+00. Their $J-H$ and $H-K$ colors are also similar to the other eight IR-faint stars in our sample. Our model fits to these eight stars imply mixed $\mathrm{H} / \mathrm{He}$ compositions. Therefore, based on the near-infrared colors, the newly observed ultracool WDs are likely to have mixed compositions as well.

Figure 16 presents our model fits to the SEDs of the five WDs from the Gates et al. (2004) sample assuming pure hydrogen and mixed $\mathrm{H} / \mathrm{He}$ atmosphere compositions. The SED for J0854+3503 is different from the other four stars, and our best-fit model implies a temperature above $4000 \mathrm{~K}$. Admittedly, the best-fit model is not a very good fit to the data and there is clearly something at odds with this object. The best-fit solution has $T_{\text {eff }}=4070 \mathrm{~K}$ and $\log (\mathrm{He} / \mathrm{H})=-0.95$, implying that J0854+3503 may not be an ultracool WD afterall. The unusual SED may be due to a binary WD system and parallax observations will be helpful in understanding the nature of this system.

The SEDs for the remaining four stars in the Gates et al. (2004) sample, J0947+4459, J1001+3903, J1220+0914, and $\mathrm{J} 1403+4533$, are similar to the SEDs for LHS 3250 and SDSS $1337+00$. The best-fit pure hydrogen models predict strong absorption bumps in the near-infrared including a strong absorption feature at $0.8 \mu \mathrm{m}$, which is not observed in any of these targets. Pure helium models also fail to reproduce the SEDs as these stars all show significant absorption in the optical and infrared. The solid lines in Figure 16 show the fits using mixed $\mathrm{H} / \mathrm{He}$ atmosphere models. The best-fit model temperatures are 2670-3410 K with $\log (\mathrm{H} / \mathrm{He})=-2.7$ to -5.1 . These are similar to the best-fit model solutions for LHS 1402, LHS 3250, and SDSS J1337+00.

Figure 17 presents our model fits to the SEDs of two ultracool WD candidates from Harris et al. (2008) and one from Vidrih et al. (2007). The observed optical and near-infrared SEDs of J0310-0110 and J1452+4522 are best explained with pure helium atmosphere models with $T_{\text {eff }}=4970$ and $5780 \mathrm{~K}$ and that of $\mathrm{J} 2239+0018 \mathrm{~B}$ is best explained as a pure hydrogen atmosphere WD with $T_{\text {eff }}=4440 \mathrm{~K}$. J0310-0110 and $\mathrm{J} 1452+4522$ were classified as ultracool based on the SDSS photometry and spectroscopy. However, our near-infrared observations show that these two stars do not display infrared flux deficits, and they are relatively warm WDs. Our model fit for $\mathrm{J} 1452+4522$ is unusual in the sense that the observations behave like there is an additional opacity source in the UV, although we are not aware of similar objects. Using mixed $\mathrm{H} / \mathrm{He}$ models does not improve the fits. The best-fit mixed atmosphere model has $T_{\text {eff }}=4730 \mathrm{~K}$ and $\log (\mathrm{H} / \mathrm{He})=-4.8$ (assuming $\log g=8$ ), but this model significantly over(under)-predicts the $J(K)$ band photometry. J2239+0018B is a common proper motion companion to the ultracool WD candidate J2239+0018A. Vidrih et al. (2007) identified J2239+0018B as an ultracool WD based on $K$-band photometry from the UKIDSS survey. Our observations in the $J H$ bands, UKIDSS photometry in the $K$-band, and a detailed model atmosphere analysis demonstrate that $\mathrm{J} 2239+0018 \mathrm{~B}$ is not an ultracool WD.

Harris et al. (2008) report narrow $\mathrm{H} \alpha$ (and possibly $\mathrm{H} \beta$, see Figure 17) emission in the SDSS spectrum of J0310-0110 indicating the presence of a substellar companion. Our model fit for this star is consistent with a cool He-atmosphere WD with no sign of a companion. Based on our age and distance estimates of $6.1 \mathrm{Gyr}$ and $117 \mathrm{pc}, \mathrm{J} 0310-0110$ has $M_{\mathrm{K}}=$ $13.2 \mathrm{mag}$. The $3 \sigma$ limit of the $K$-band photometry limits possible companions to fainter than about $M_{\mathrm{K}}=16.3 \mathrm{mag}$. At $5 \mathrm{Gyr}$ of age, a $0.05 M_{\odot}$ companion would have an absolute magnitude of $M_{\mathrm{K}}=16.4$ mag (Baraffe et al. 2003). Thus, if J0310-0110 has a companion, it must be less massive than about $0.05 M_{\odot}$.

Figure 18 presents our model fits to the SEDs of the remaining five stars from the Harris et al. (2008) sample. The observed SEDs for these stars are best fit with mixed $\mathrm{H} / \mathrm{He}$ atmosphere models with $T_{\text {eff }}=2290-4160 \mathrm{~K}$ and $\log (\mathrm{H} / \mathrm{He})=$ -2.3 to -7.8 . While these fits are better than the fits using pure hydrogen or pure helium models, they are not perfect. Like LHS 1402, LHS 3250, and SDSS J1337+00, the predictions of the peaks of the energy distributions of the ultracool WDs in our sample (Figures 16 and 18) are too sharp compared with the observations. The best-fit model temperature for $\mathrm{J} 1238+3502$ is unusually low $(2290 \mathrm{~K})$ and the required helium abundance is relatively high. However, only $J$-band observations are available in the infrared, and our model fit parameters may be improved with additional near-infrared observations (although such observations are difficult to obtain since J1238+3502 is relatively faint in the near-infrared). The poor model fit for $\mathrm{J} 1251+4403$ is similar to that of $\mathrm{J} 1403+4533$; the observations peak at a bluer wavelength compared with the best-fit model. The $u$ - and $g$-band photometry suggests very cool $(\sim 2000 \mathrm{~K})$ WDs, but the riz and infrared photometry requires a hotter WD. This is probably an indication that the CIA opacities are wrong at such low temperatures. Despite the fact that our current model atmospheres do not find perfect fits to the observed photometry, we can rule out extreme hydrogen-rich compositions for these stars based on the current CIA opacity calculations.

\section{DISCUSSION}

\subsection{Chemical Abundance Patterns}

Our detailed model atmosphere analysis of 126 cool WDs with optical and near-infrared photometry shows that 61 stars (48\%) have pure hydrogen, 44 stars $(35 \%)$ have pure helium 

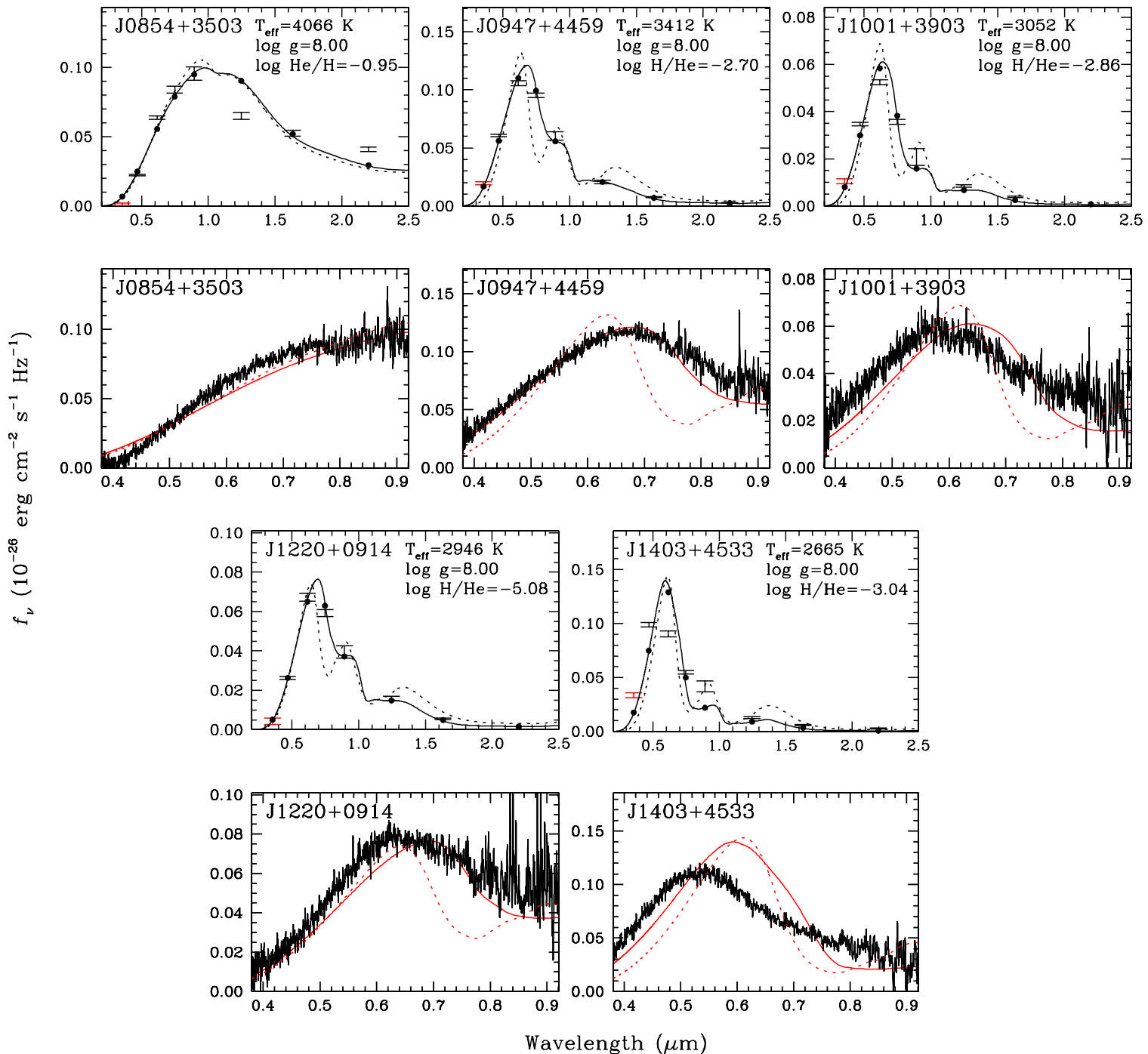

Figure 16. Fits to the SEDs of the ultracool WDs discovered by Gates et al. (2004) using pure hydrogen (dotted lines) and mixed H/He (solid lines) atmosphere models. The top panels show the optical and near-infrared photometry, whereas the bottom panels show the SDSS spectrum of each object.

(A color version of this figure is available in the online journal.)
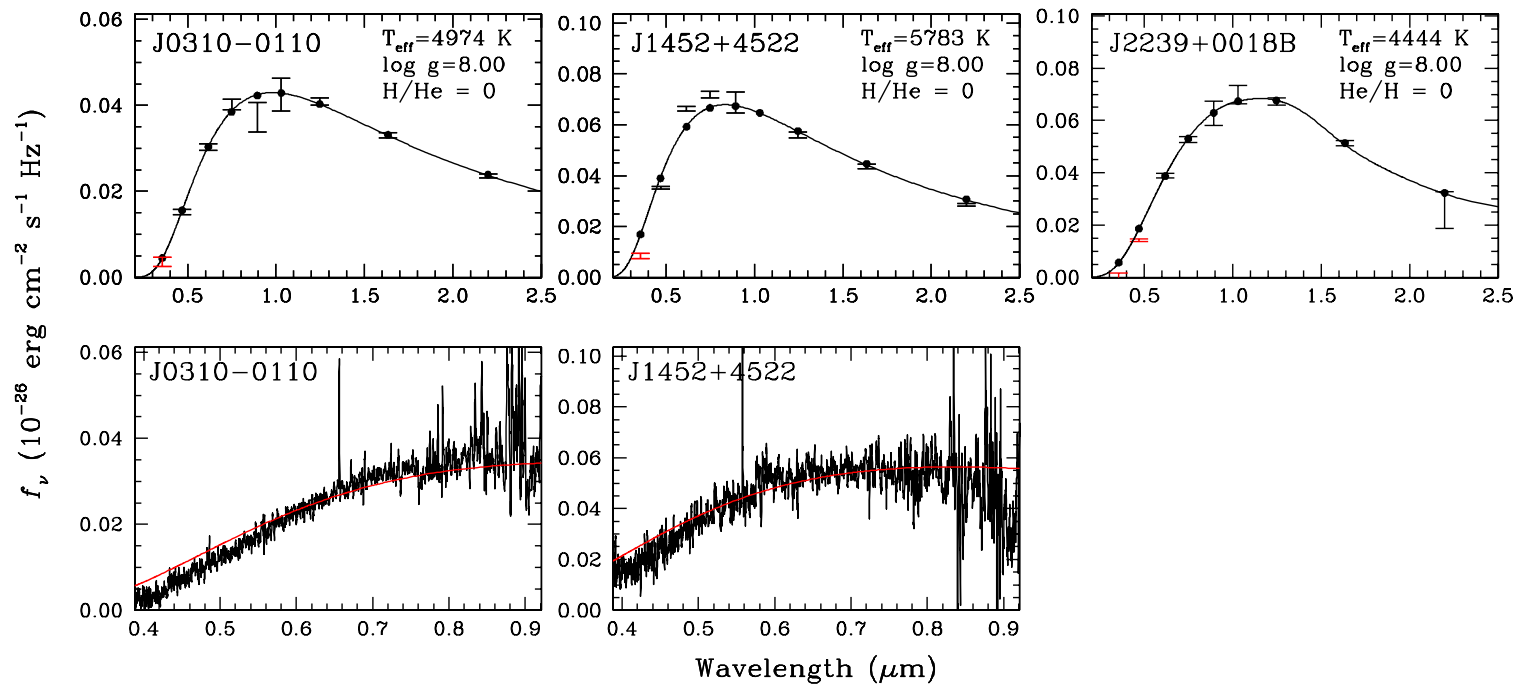

Figure 17. Similar to Figure 16, but for three ultracool WD candidates from Harris et al. (2008). Our model fits show that J0310-0110 and J1452+4522 are best explained as pure helium atmosphere WDs and J2239+0018B as a pure hydrogen atmosphere WD. The best-fit models have temperatures above 4000 K, implying that these three stars are not ultracool.

(A color version of this figure is available in the online journal.) 

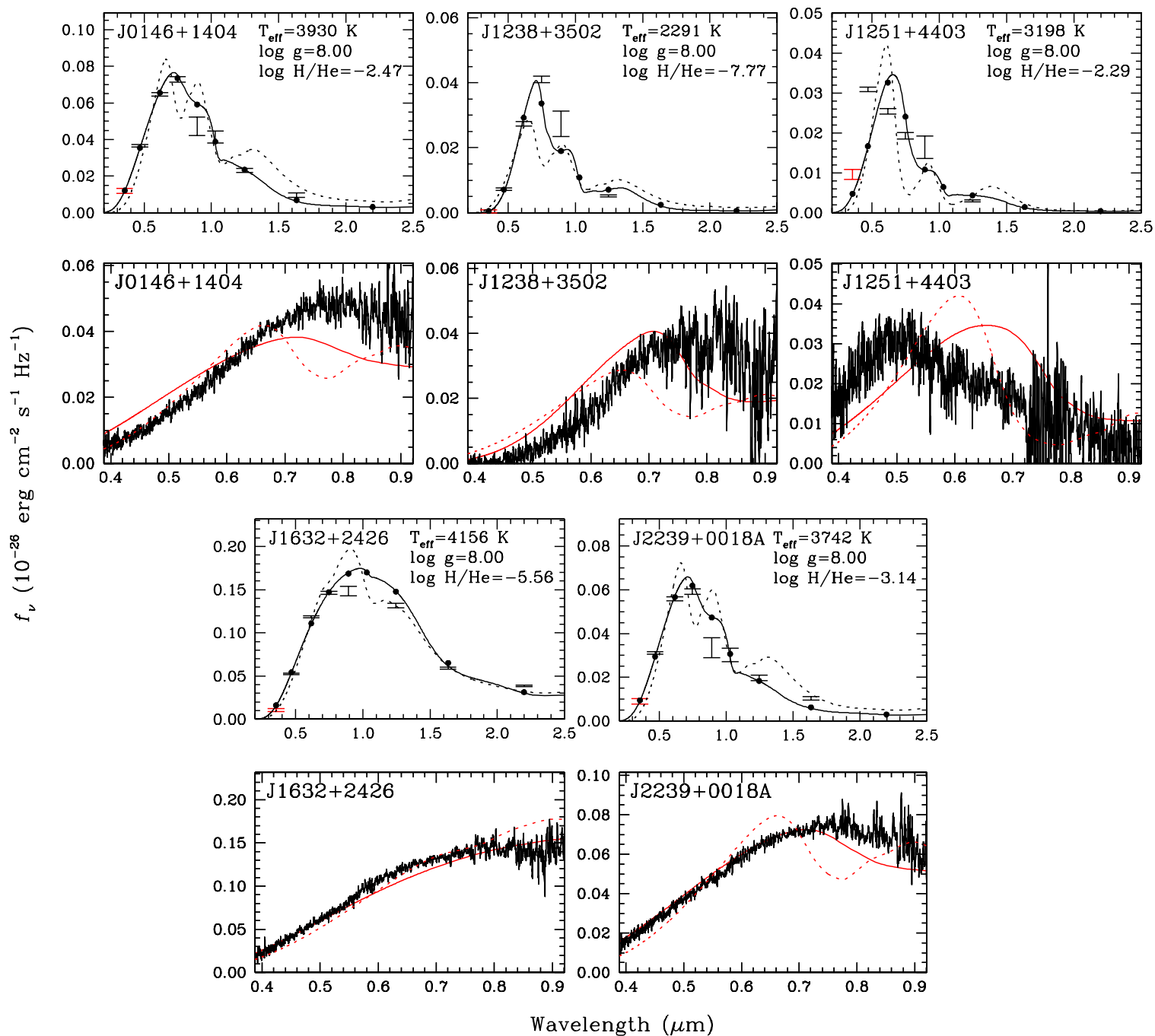

Figure 18. Similar to Figure 16, but for five ultracool WD candidates from Harris et al. (2008). All five stars are best fit with mixed H/He atmosphere models (solid lines).

(A color version of this figure is available in the online journal.)

(including DQ WDs with helium-dominated atmospheres), and 21 stars have mixed $\mathrm{H} / \mathrm{He}$ atmospheres. The latter include 10 ultracool WD candidates and 2 peculiar DQ WDs. Based on a detailed model atmosphere analysis of 150 WDs with $T_{\text {eff }} \leqslant$ $12,000 \mathrm{~K}$, Bergeron et al. (2001) find the frequency of pure hydrogen and pure helium atmosphere WDs to be $64 \%$ and $33 \%$, respectively.

Figure 19 presents the surface composition measurements for our sample and the Bergeron et al. (2001) sample of WDs. Bergeron et al. (2001) find helium-rich atmosphere WDs down to about $4500 \mathrm{~K}$ and hydrogen-rich WDs down to $4000 \mathrm{~K}$. The coolest and oldest WDs are likely to accrete from the interstellar medium in their $\sim 10 \mathrm{Gyr}$ lifetimes. The lack of pure helium WDs below $4500 \mathrm{~K}$ supports this scenario. Bergeron et al. (2001) also find a non-DA gap (or a deficiency in number) between about $5000 \mathrm{~K}$ and $6000 \mathrm{~K}$. They find non-DA stars below and above this temperature range, but they find only three non-DA stars in the gap. In addition, they do not find a large population of mixed $\mathrm{H} / \mathrm{He}$ atmosphere WDs. In contrast, our sample is restricted to stars cooler than about $6600 \mathrm{~K}$, and the fraction of mixed $\mathrm{H} / \mathrm{He}$ atmosphere WDs is larger. Our sample fills in the non-DA gap somewhat. However, there is still a gap between $5600 \mathrm{~K}$ and $6200 \mathrm{~K}$ in both our and the Bergeron et al. (2001) sample (though a selection bias is evident in our sample, which has only a few stars warmer than $6000 \mathrm{~K}$ ). None of our WD targets identified as pure helium atmosphere objects with $T_{\text {eff }}=5400-5600 \mathrm{~K}$ have optical spectroscopy available. Given the slight differences between the pure hydrogen and pure helium model SEDs for this temperature range, our pure helium classification based on the SED fits should be taken with caution. Therefore, the non-DA gap may extend down to $5400 \mathrm{~K}$. Perhaps another important piece of evidence for the existence of this non-DA gap comes from the work by Kilic et al. (2006a) who identified five DCs in the non-DA gap. So far four of these DCs are observed in the near-infrared (in this work and also in Kilic et al. 2009), and it turns out that all four stars have mixed $\mathrm{H} / \mathrm{He}$ atmospheres. The SEDs of three of these stars, J0157+1335, J1648+3939, and $\mathrm{J} 1722+5752$, are shown in Figure 14. In addition, J1203+0426 is identified as a mixed $\mathrm{H} / \mathrm{He}$ atmosphere WD by Kilic et al. (2009). Our model fits with mixed $\mathrm{H} / \mathrm{He}$ atmospheres place these stars outside of the non-DA gap.

Our model fits imply that a significant fraction of WDs in the temperature range $4500-5000 \mathrm{~K}$ are $\mathrm{He}$ rich. Since $\mathrm{H} \alpha$ is invisible at these temperatures, the choice of composition depends on the quality of the fits to the SEDs. The best H-rich 


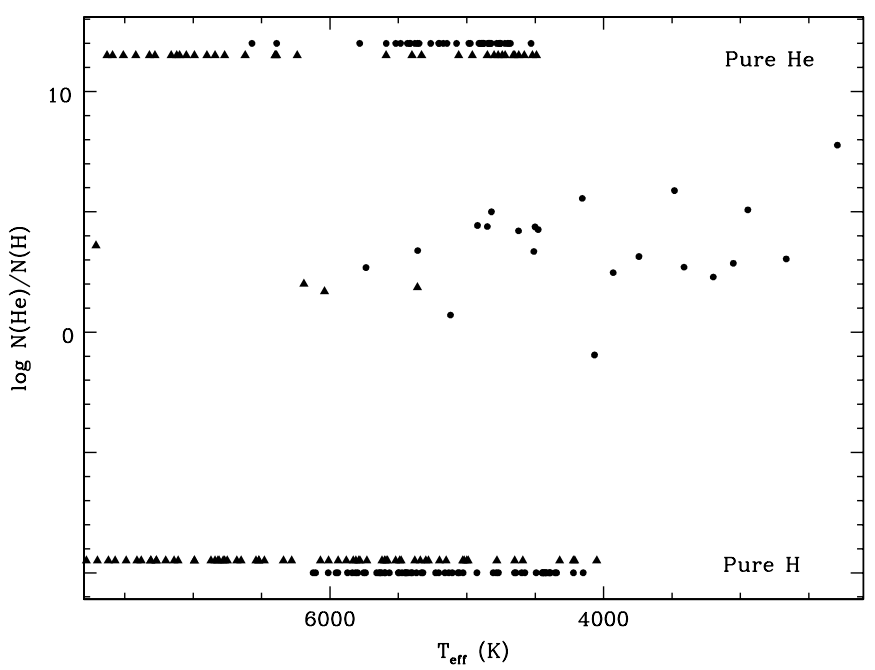

Figure 19. Distribution of hydrogen and helium abundances for our sample of cool WDs (circles) and that of Bergeron et al. (2001, triangles).

model fit is sometimes not too different from the He-rich model fit. It is possible that small shifts in the ugriz and $J H K$ calibration may explain the overabundance of He-rich objects in this temperature range. However, a similar number distribution is also evident in the Bergeron et al. (2001) sample, which relies on $B V R I$ and $J H K$ photometry. The non-inclusion of the Ly $\alpha$ opacity in our models or problems with the CIA calculations may cause incorrect assignment of the atmospheric types to WDs in this temperature range and further work is required to understand if the observed overabundance of $\mathrm{He}$ rich atmosphere WDs at this temperature range is real.

Overall, our analysis reveals a complex spectral evolutionary history for cool WDs (see also Bergeron et al. 2001). The model atmosphere analysis by Kowalski \& Saumon (2006) presents a completely different picture, in which WDs below $6000 \mathrm{~K}$ are hydrogen rich. They come to this conclusion by excluding the DQ and DZ WDs from their sample and also using a different set of pure helium atmosphere models that have colors essentially the same as blackbodies. Since the cool WD SEDs are not blackbodies, they assign hydrogen-rich composition for most cool WDs and they propose a simple evolutionary scenario in which WDs accrete hydrogen from the interstellar medium and turn into hydrogen-rich WDs even if they start with pure helium atmospheres. Our pure helium atmosphere models have colors slightly different from simple blackbodies (see Figure 8) and more similar to the observed colors of cool DC WDs. Resolving the discrepancy between our interpretation and the Kowalski \& Saumon (2006) results requires a thorough study of the differences between these models. DZ WDs are the only cool $\left(T_{\text {eff }}<5000 \mathrm{~K}\right)$ WDs with atomic absorption lines. A detailed model atmosphere analysis of cool DZs (e.g., Dufour et al. 2007) would be a crucial test for identifying problems with both sets of models.

\subsection{Stellar Ages}

Our sample is complete for the faint end of the luminosity function $\left(M_{\mathrm{bol}} \geqslant 14.6 \mathrm{mag}\right.$ ) from Harris et al. (2006, although this luminosity function is limited to $V_{\tan } \geqslant 30 \mathrm{~km} \mathrm{~s}^{-1}$ objects and is not complete itself) and it can be used to constrain the age of the Galactic disk. WD cooling rates depend on the radius (mass) of the star. Without a parallax measurement, we cannot reliably determine the radii and masses of our targets,

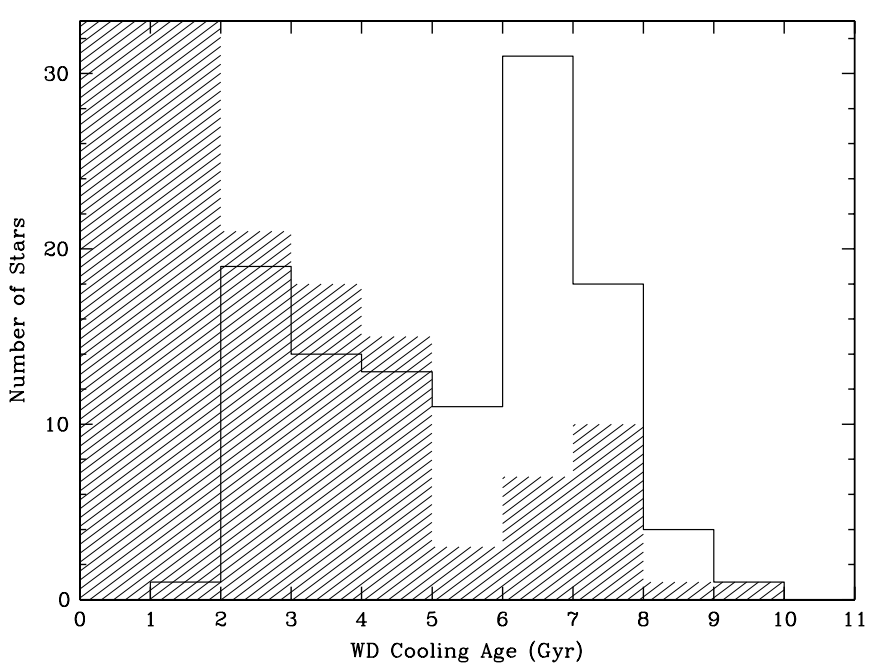

Figure 20. Age distribution of our sample of WDs (solid histogram, excluding the ultracool WD candidates due to poor model fits) compared with that of Bergeron et al. (2001, shaded histogram).

and hence ages. However, the average mass for the 48 WDs cooler than $6000 \mathrm{~K}$ in the Bergeron et al. (2001) sample of WDs with parallax measurements is $0.61 M_{\odot}$. Therefore, our assumption of $\log g=8$ is reasonable. Based on our model fits, the implied cooling ages and distances for our sample are given in Table 3. The predicted distances range from $15 \mathrm{pc}$ to $130 \mathrm{pc}$. Given the uncertainties in the distance estimates, several targets may be part of the $20 \mathrm{pc}$ sample. The closest star in our sample is $\mathrm{J} 2325+1403$, predicted to be at 15.4 pc. Lépine et al. (2009) present trigonometric parallax observations of $\mathbf{J} 2325+1403$, which indicate that it is at $22 \mathrm{pc}$. Using this distance measurement in our model fits, J2325+1403 has $T_{\text {eff }}=5070 \mathrm{~K}$ and $\log g=7.39$. Not surprisingly, its mass $\left(0.28 \pm 0.03 M_{\odot}\right)$ has to be significantly lower than the average $\left(\log g=8\right.$ or $\left.M=0.58 M_{\odot}\right)$ to match the distance. Alternatively, J2325+1403 could be an unresolved double degenerate binary since it is overluminous.

The estimated WD cooling ages for our sample range from 1.8 Gyr to 12.1 Gyr. Excluding the ultracool WDs (due to poor model fits and relatively uncertain ages, Bergeron \& Leggett 2002) and J2242+0048 (with strong infrared absorption), the coolest WDs in our sample have temperatures around $4200 \mathrm{~K}$ (see Figure 13). This is comparable to the coolest WDs in the Bergeron et al. (2001) sample. Figure 20 shows the age distribution of our sample of WDs (excluding the ultracool WD candidates) compared with that of Bergeron et al. (2001) sample, which also includes the majority of the objects from the Leggett et al. (1998) WD luminosity function. Compared with the Bergeron et al. (2001) sample, we have a significantly larger number of stars older than 5 Gyr. Both samples show a significant drop in the number of stars at 8 Gyr. Even though the individual ages for our targets cannot be trusted due to the unknown masses, the average mass for our sample should be about $0.6 M_{\odot}$ and the average age for the oldest stars in our sample should be reliable. Adding 1.4 Gyr for the mainsequence lifetime of the $2 M_{\odot}$ solar-metallicity progenitor stars (Marigo et al. 2008) brings the total age to about 9.4 Gyr, entirely consistent with the oldest WDs in Table 2 of Leggett et al. (1998) and the Galactic disk age of $8 \pm 1.5 \mathrm{Gyr}$.

There are four common proper motion WD pairs in our sample. These systems provide a consistency check in our distance and age measurements. Two of these systems, J0947+4459 


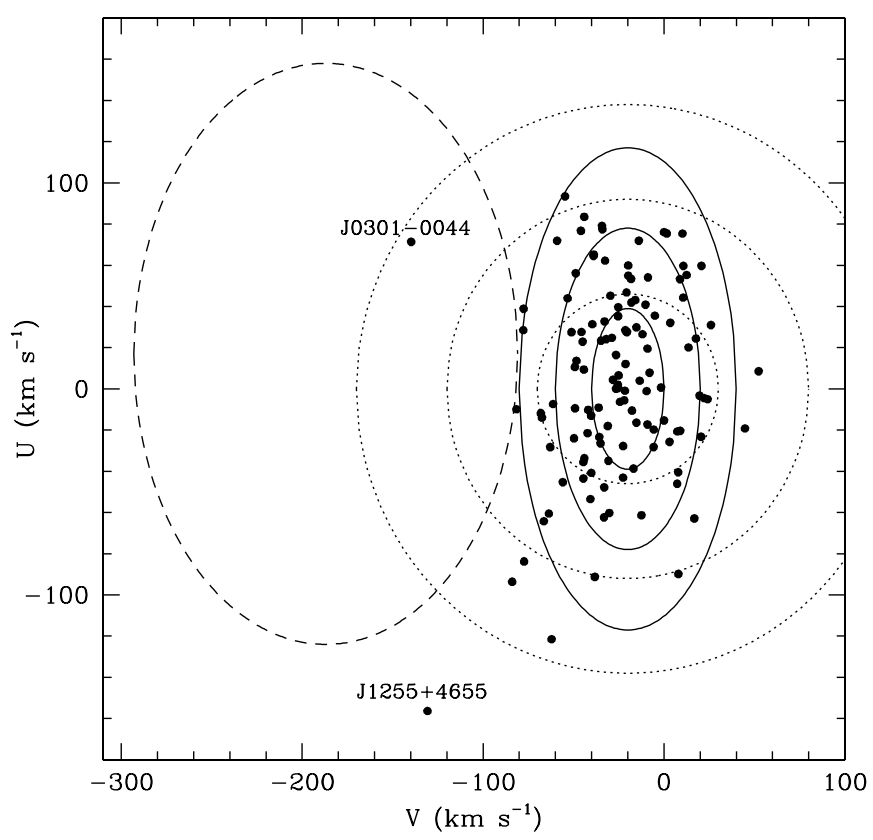

Figure 21. $U$ vs. $V$ velocities for our targets assuming $\log g=8.0$ and zero radial velocity. The $1 \sigma, 2 \sigma$, and $3 \sigma$ velocity ellipses of the thin disk (solid line) and the thick disk (dotted line), and the $1 \sigma$ ellipse of the halo (dashed line) are also shown.

and $\mathbf{J} 2239+0018$, include ultracool WDs and are discussed in Section 4.4. We derive distances of 52 and 54 pc and cooling ages of 2.6 and $6.3 \mathrm{Gyr}$ for the two WDs in the J0747+2438 system, respectively. The difference in cooling ages can be explained by a small mass difference (of order $0.3 M_{\odot}$ ) between the two stars. We derive distances of 51 and $82 \mathrm{pc}$ and cooling ages of 2.6 and $7.8 \mathrm{Gyr}$ for the two WDs in the J2321+0102 system. However, these differences can also be explained by a small mass difference between the two stars in the system. Parallax measurements will be useful to constrain the individual masses in these systems.

\subsection{Disk or Halo Membership}

Bergeron et al. (2005) demonstrate the importance of determining total stellar ages in order to associate any WD with the thick disk or halo. Given the total estimated ages of $<10 \mathrm{Gyr}$, the majority of the objects in our sample most likely belong to the disk population. Figure 21 shows the Galactic UV velocities of our targets (based on the model fits assuming $\log g=8$ ) compared to the velocity ellipses of the disks and the halo (Chiba \& Beers 2000). All but two of the objects in our sample, including the ultracool WDs, have velocities consistent with the thin disk or thick disk objects. Reid (2005) argue that the thick disk is likely to contribute about $20 \%$ of the solar neighborhood WDs, and these WDs should dominate at faint absolute magnitudes. About $27 \%$ of our targets have UV velocities inconsistent with the thin disk population at $2 \sigma$, and these objects may belong to the thick disk. However, this fraction is very uncertain due to the lack of parallax measurements.

J0301-0044 and J1255+4655 have UV velocities that are inconsistent with the disk population at more than $2 \sigma$, and they are halo WD candidates. Both have $T_{\text {eff }} \approx 4500 \mathrm{~K}$ with WD cooling ages of 7.1-7.3 Gyr and total ages $\approx 8$ Gyr for average mass WDs. These ages are relatively young for halo objects. However, a slightly lower mass around $0.5 M_{\odot}$ would make the total ages for J0301-0044 and J1255+4655 consistent with the halo population.
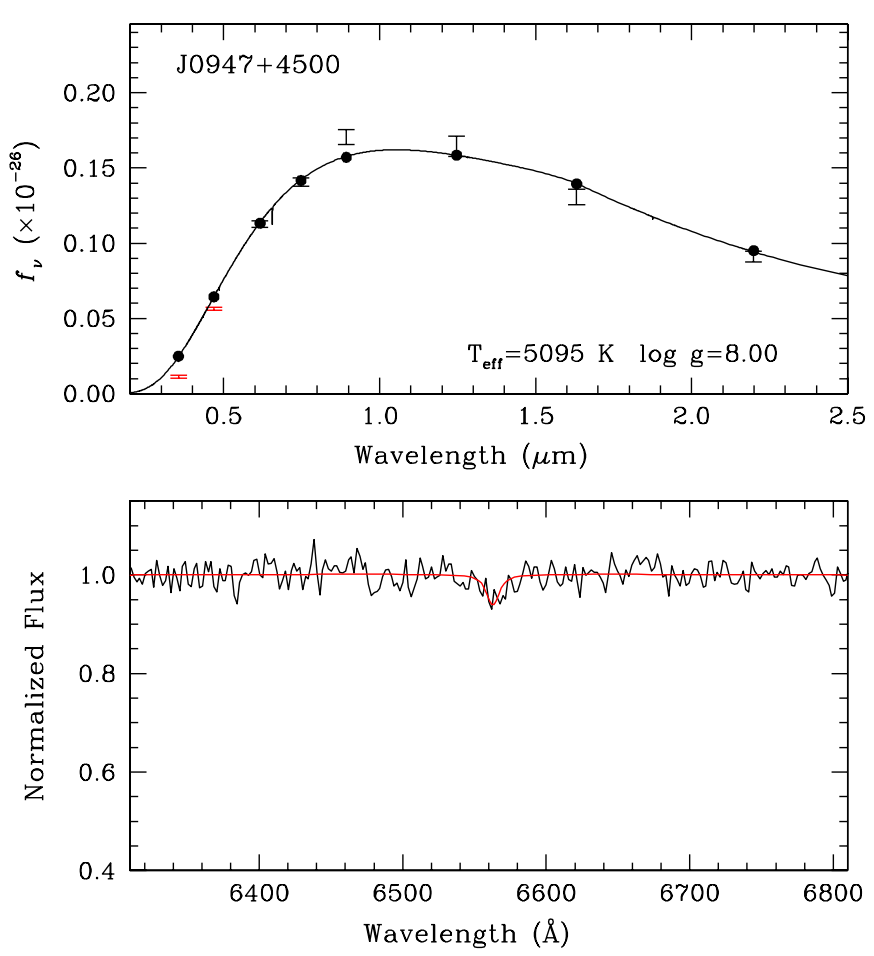

Figure 22. Companion to the ultracool WD J0947+4459.

(A color version of this figure is available in the online journal.)

\subsection{Ultracool WDs}

Our model fits to the SEDs of ultracool WDs are not perfect, but the best-fit models imply mixed $\mathrm{H} / \mathrm{He}$ atmosphere composition. The near-infrared colors of ultracool WDs are similar to the relatively warmer WDs that also show flux deficits in the near-infrared. In addition, the observed WD sequence in $r-J$ versus $J-H$ color-color diagram suggests that the ultracool WD sequence is an extension of the warmer WDs with near-infrared flux deficits. Since our mixed $\mathrm{H} / \mathrm{He}$ atmosphere models fit the SEDs of warmer WDs with flux deficits fairly well, there is a high probability that the ultracool WDs also have mixed $\mathrm{H} / \mathrm{He}$ atmospheres. Based on our model fits with $\log g=8$, we estimate that the 10 ultracool WD candidates presented in Figures 16 and 18 are 24-66 pc away and they have WD cooling ages in the range 8.0-12.1 Gyr. These would correspond to total ages (including main-sequence lifetimes) of $\approx 9-13$ Gyr. The implied tangential velocities are in the range $16-92 \mathrm{~km} \mathrm{~s}^{-1}$, indicating that these ultracool WDs most likely belong to the thick disk population.

Trigonometric parallax measurements are available for only one ultracool WD so far. The distance measurement of $30.3 \pm 0.5 \mathrm{pc}$ for LHS 3250 (Harris et al. 1999) implies that it is brighter than expected for a $0.6 M_{\odot} \mathrm{WD}$, unless it is warmer by about $700 \mathrm{~K}$. Given the similarities between the SEDs of LHS 3250 and the ultracool WDs in our sample, it is likely that these WDs are also brighter, more distant, and younger than our model fits with $\log g=8$ imply. In fact, our preliminary parallax observations at the MDM $2.4 \mathrm{~m}$ telescope for a few of these ultracool WDs show that the distances are underestimated in our model fits. Hence, they are likely to be less massive than $0.6 M_{\odot}$ (perhaps a binary origin) or hotter than currently predicted from the model fits. Further improvements in the CIA opacity and model atmosphere calculations may help in matching the observed SEDs and luminosities. Until then, the nature of these stars remains uncertain. 
Two of the ultracool WDs, J0947+4459 and J2239+0018, have common proper motion companions. The model fits to the $\mathrm{J} 2239+0018 \mathrm{~A} / \mathrm{B}$ system are rather good and the ages (8.9 and $7.8 \mathrm{Gyr})$ and distances (60 and $79 \mathrm{pc})$ agree reasonably well. This is encouraging because one of the components is a mixed $\mathrm{H} / \mathrm{He}$ atmosphere WD and the other is a very cool H-rich WD. For an average mass WD, J2239+0018B has a total age (WD cooling + main-sequence lifetime) of about $9.2 \mathrm{Gyr}$.

The optical spectrum of the companion for J0947+4459 displays weak $\mathrm{H} \alpha$ absorption. Figure 22 shows our pure hydrogen atmosphere model fits to the J0947+4500 SED. Omitting the $u$ - and $g$-band photometry, the best-fit temperature is $5095 \pm$ $130 \mathrm{~K}$. This corresponds to a distance of $61 \mathrm{pc}$ and a WD cooling age of 5.2 Gyr. The observed $\mathrm{H} \alpha$ absorption is consistent with the model prediction. The ultracool WD J0947+4459 has an estimated distance of $39 \mathrm{pc}$ and an age of $9.5 \mathrm{Gyr}$. If the ultracool WD is slightly lower mass or hotter, it would be more distant (at $61 \mathrm{pc}$ ), brighter, and its implied WD cooling age would be similar to its companion. Of course, all of these can be resolved when accurate parallax measurements are available.

\section{CONCLUSIONS}

We present follow-up optical spectroscopy and near-infrared photometry of the cool and ultracool WDs in the SDSS DR3 that are identified by Kilic et al. (2006a), Gates et al. (2004), and Harris et al. (2006, 2008). We demonstrate that a clean selection of WDs is possible using a reduced proper motion diagram and a tangential velocity cut-off of $30 \mathrm{~km} \mathrm{~s}^{-1}$. This can be used to select large samples of cool WDs from the SDSS DR7 and SEGUE data or any other survey that uses the SDSS filter set.

Our near-infrared observations reveal eight new stars with significant absorption. All of these stars are best explained with mixed $\mathrm{H} / \mathrm{He}$ atmosphere models. The infrared colors of ultracool WDs are similar to these eight stars as well. Hence, there is indirect evidence that they also have mixed $\mathrm{H} / \mathrm{He}$ atmospheres. Our model fits to the ultracool WD SEDs show that they may be as cool as $\approx 2300 \mathrm{~K}$ and as old as $13 \mathrm{Gyr}$ (including the main-sequence age of $1 \mathrm{Gyr}$ for a $2 M_{\odot}$ thick disk star). Their implied tangential velocities and Galactic space velocities are consistent with the thick disk objects. Further progress in understanding the ultracool WDs and estimating reliable temperatures, masses, and ages can be achieved with the help of trigonometric parallax observations. Such observations for the ultracool WDs identified by Gates et al. (2004) and Harris et al. (2008) are currently underway at the MDM $2.4 \mathrm{~m}$ and the USNO $1.55 \mathrm{~m}$ telescopes.

Only two objects in our sample have space velocities consistent with the halo population. Trigonometric parallax observations for these targets will be required to confirm their halo membership. The absence of more halo WD candidates in our sample is not surprising as our survey is limited to objects brighter than about $g=19.5 \mathrm{mag}$ due to the brightness limit of the Palomar Observatory Sky Survey plates. A deeper, wide-field astrometric survey is currently being conducted at the Bok $90^{\prime \prime}$ and USNO $1.3 \mathrm{~m}$ telescopes (Liebert et al. 2007; Munn et al. 2010). Initial follow-up observations of candidates from this survey are already finding the elusive old halo WD candidates in the solar neighborhood (Kilic et al. 2010). This survey, along with the Pan-Starrs and LSST surveys, will be an invaluable resource for halo WD studies.

We thank J. Liebert for many useful discussions, Andy Stephens for the derivation of the NIRI nonlinearity correction, and an anonymous referee for useful suggestions. Support for this work was provided by NASA through the Spitzer Space Telescope Fellowship Program, under an award from Caltech. This material is also based on work supported by the National Science Foundation under grants AST-0607480 and AST-0602288 and by the NSERC Canada and by the Fund FQRNT (Québec). S.K.L.'s research is supported by Gemini Observatory. P. Bergeron is a Cottrell Scholar of Research Corporation for Science Advancement.

The Hobby-Eberly Telescope (HET) is a joint project of the University of Texas at Austin, The Pennsylvania State University, Stanford University, Ludwig-Maximilians-Universitat Munchen, and Georg-August-Universitat Gottingen. The HET is named in honor of its principal benefactors, William P. Hobby and Robert E. Eberly. The Gemini observatory is operated by the Association of Universities for Research in Astronomy, Inc., under a cooperative agreement with the NSF on behalf of the Gemini partnership: the National Science Foundation (United States), the Science and Technology Facilities Council (United Kingdom), the National Research Council (Canada), CONICYT (Chile), the Australian Research Council (Australia), Ministério da Ciência e Tecnologia (Brazil), and Ministerio de Ciencia, Tecnología e Innovación Productiva (Argentina). The IRTF is operated by the University of Hawaii under Cooperative Agreement no. NCC 5-538 with the National Aeronautics and Space Administration, Office of Space Science, Planetary Astronomy Program.

\section{REFERENCES}

Baraffe, I., Chabrier, G., Barman, T. S., Allard, F., \& Hauschildt, P. H. 2003, A\&A, 402, 701

Bergeron, P., \& Leggett, S. K. 2002, ApJ, 580, 1070

Bergeron, P., Leggett, S. K., \& Ruiz, M. T. 2001, ApJS, 133, 413

Bergeron, P., Ruiz, M. T., Hamuy, M., Leggett, S. K., Currie, M. J., Lajoie, C.-P., \& Dufour, P. 2005, ApJ, 625, 838

Bergeron, P., Ruiz, M. T., \& Leggett, S. K. 1997, ApJS, 108, 339

Bergeron, P., Ruiz, M.-T., Leggett, S. K., Saumon, D., \& Wesemael, F. 1994, ApJ, 423, 456

Bergeron, P., Saumon, D., \& Wesemael, F. 1995, ApJ, 443, 764

Carollo, D., Koester, D., Spagna, A., Lattanzi, M. G., \& Hodgkin, S. T. 2003, A\&A, 400, L13

Carollo, D., et al. 2006, A\&A, 448, 579

Chiba, M., \& Beers, T. C. 2000, AJ, 119, 2843

Dufour, P., Bergeron, P., \& Fontaine, G. 2005, ApJ, 627, 404

Dufour, P., et al. 2007, ApJ, 663, 1291

Eisenstein, D. J., et al. 2006, ApJS, 167, 40

Fontaine, G., Brassard, P., \& Bergeron, P. 2001, PASP, 113, 409

Gates, E., et al. 2004, ApJ, 612, 129

Hall, P. B., \& Maxwell, A. J. 2008, ApJ, 678, 1292

Hambly, N. C., Smartt, S. J., Hodgkin, S. T., Jameson, R. F., Kemp, S. N., Rolleston, W. R. J., \& Steele, I. A. 1999, MNRAS, 309, L33

Harris, H. C., Dahn, C. C., Vrba, F. J., Henden, A. A., Liebert, J., Schmidt, G. D., \& Reid, I. N. 1999, ApJ, 524, 1000

Harris, G. J., Lynas-Gray, A. E., Miller, S., \& Tennyson, J. 2004, ApJ, 617, L143

Harris, H. C., et al. 2001, ApJ, 549, L109

Harris, H. C., et al. 2006, AJ, 131, 571

Harris, H. C., et al. 2008, ApJ, 679, 697

Hill, G. J., Nicklas, H. E., MacQueen, P. J., Tejada, C., Cobos Duenas, F. J., \& Mitsch, W. 1998, Proc. SPIE, 3355, 375

Hintzen, P. 1986, AJ, 92, 431

Hogg, D. W. 2001, AJ, 122, 2129

Holberg, J. B., \& Bergeron, P. 2006, AJ, 132, 1221

Hummer, D. G., \& Mihalas, D. 1988, ApJ, 331, 794

John, T. L. 1994, MNRAS, 269, 871

Kilic, M., Kowalski, P. M., Mullally, F., Reach, W. T., \& von Hippel, T. 2008, ApJ, 678, 1298

Kilic, M., Kowalski, P. M., \& von Hippel, T. 2009, AJ, 138, 102

Kilic, M., von Hippel, T., Méndez, R. A., \& Winget, D. E. 2004, ApJ, 609, 766 
Kilic, M., von Hippel, T., Mullally, F., Reach, W. T., Kuchner, M. J., Winget, D. E., \& Burrows, A. 2006, ApJ, 642, 1051

Kilic, M., et al. 2006, AJ, 131, 582

Kilic, M., et al. 2010, ApJ, 715, L21

Kleinman, S. J., et al. 2004, ApJ, 607, 426

Kowalski, P. M. 2006, ApJ, 641, 488

Kowalski, P. M., \& Saumon, D. 2006, ApJ, 651, L137

Lawrence, A., et al. 2007, MNRAS, 379, 1599

Leggett, S. K., Ruiz, M. T., \& Bergeron, P. 1998, ApJ, 497, 294

Leggett, S. K., et al. 2006, MNRAS, 373, 781

Lépine, S., Thorstensen, J. R., Shara, M. M., \& Rich, R. M. 2009, AJ, 137, 4109

Liebert, J., Dahn, C. C., \& Monet, D. G. 1988, ApJ, 332, 891

Liebert, J., Kilic, M., Williams, K. A., von Hippel, T., Winget, D. M. J., Harris, H. C., Levine, S., \& Metcalfe, T. S. 2007, in ASP Conf. Ser. 372, 15th European Workshop on White Dwarfs, ed. R. Napiwotzki \& M. R. Burleigh (San Francisco, CA: ASP), 129

Lodieu, N., Leggett, S. K., Bergeron, P., \& Nitta, A. 2009, ApJ, 692, 1506

Luyten, W. J. 1979, LHS Catalogue (2nd ed.; Minneapolis: Univ. of Minnesota) Marigo, P., Girardi, L., Bressan, A., Groenewegen, M. A. T., Silva, L., \& Granato, G. L. 2008, A\&A, 482, 883

Monet, D. G., et al. 2003, AJ, 125, 984
Munn, J. A., DeGennaro, S., Harris, H., von Hippel, T., Jeffery, E., Kilic, M., Liebert, J., \& Williams, K. 2010, BAAS, 42, 461

Munn, J. A., et al. 2004, AJ, 127, 3034

Oppenheimer, B. R., Hambly, N. C., Digby, A. P., Hodgkin, S. T., \& Saumon, D. 2001, Science, 292, 698

Oswalt, T. D., Smith, J. A., Wood, M. A., \& Hintzen, P. 1996, Nature, 382, 692

Pier, J. R., et al. 2003, AJ, 125, 1559

Pickles, A. J. 1998, PASP, 110, 863

Reid, I. N. 2005, ARA\&A, 43, 247

Schmidt, G. D., Bergeron, P., \& Fegley, B. 1995, ApJ, 443, 274

Sion, E. M., Greenstein, J. L., Landstreet, J. D., Liebert, J., Shipman, H. L., \& Wegner, G. A. 1983, ApJ, 269, 253

Smith, J. A., et al. 2002, AJ, 123, 2121

Tokunaga, A. T., \& Vacca, W. D. 2005, PASP, 117, 421

Tremblay, P.-E., \& Bergeron, P. 2007, ApJ, 657, 1013

Tremblay, P.-E., \& Bergeron, P. 2008, ApJ, 672, 1144

Vennes, S., \& Kawka, A. 2003, ApJ, 586, L95

Vidrih, S., et al. 2007, MNRAS, 382, 515

Winget, D. E., Hansen, C. J., Liebert, J., Van Horn, H. M., Fontaine, G., Nather, R. E., Kepler, S. O., \& Lamb, D. Q. 1987, ApJ, 315, L77 